\title{
GEOMETRY OF BANACH SPACES OF FUNCTIONS ASSOCIATED WITH CONCAVE FUNCTIONS
}

\author{
BY \\ PAUL HLAVAC( $\left.{ }^{1}\right)$ AND K. SUNDARESAN $\left({ }^{2}\right)$
}

\begin{abstract}
Let $(X, \Sigma, \mu)$ be a positive measure space, and $\phi$ be a concave nondecreasing function on $R^{+} \rightarrow R^{+}$with $\phi(0)=0$. Let $N_{\phi}(R)$ be the Lorentz space associated with the function $\phi$. In this paper a complete characterization of the extreme points of the unit ball of $N_{\phi}(R)$ is provided. It is also shown that the space $N_{\phi}(R)$ is not reflexive in all nontrivial cases, thus generalizing a result of Lorentz. Several analytical properties of spaces $N_{\phi}(R)$, and their abstract analogues $N_{\phi}(E)$, are obtained when $E$ is a Banach space.
\end{abstract}

1. Introduction. Banach spaces of measurable functions have been studied extensively by various authors in different contexts. Most of these spaces are related to classical spaces $L_{p}(\mu), 1 \leqslant p \leqslant \infty$, or else they are generalizations of these spaces. Examples of these spaces, other than Orlicz spaces, Krasnosel'skii and Rutickii [10], are the Riesz spaces, Luxemburg and Zaanen [17]. While most aspects of Orlicz spaces have been studied, it is believed that there has as yet been no systematic study of the geometric properties, or problems related to operator theory of the more general class of Riesz spaces. Most of the study of Riesz spaces is concerned with the analytical generalizations of the well-known properties of their classical counterparts. The nonexistence of a complete study is understandable because of the general nature of the definition of a Riesz space, but little is known in the directions mentioned above even concerning concrete examples of Riesz spaces. One such example is the class of Banach spaces which is called in this paper, Lorentz spaces, and their abstract analogues introduced here.

Let $(X, \Sigma, \mu)$ be a positive measure space, and $\phi$ be a concave nondecreasing function on $R^{+} \rightarrow R^{+}$with $\phi(0)=0$. Let $N_{\phi}(R)$ be the linear space of equivalence classes of measurable real valued functions defined on $X$ such that

Received by the editors August 14, 1974.

AMS (MOS) subject classifications (1970). Primary 46B10, 28A45, 46N05; Secondary 46E30, 46E40.

Key words and phrases. Lorentz spaces, vector valued functions, extreme points, function spaces, rearrangement function, vector valued measures.

(1) Part of the material in the paper is taken from the author's Doctoral Dissertation submitted to Carnegie-Mellon University (1973).

(2) The reasearch was supported in part by National Science Foundation Grant GP. 43213. 


$$
\|f\|=\int_{0}^{\infty} \phi(\mu\{x:|f(x)|>y\}) d y<\infty .
$$

With this norm $N_{\phi}(R)$ is a Banach space, Lorentz [12], Steigerwalt and White [24]. Lorentz [12] introduced a special class of these spaces with $\phi(t)=t^{\alpha}, 0<$ $\alpha<1$. In [12], Lorentz studied the space $N_{\phi}(R)$ and a generalization of this space. Halperin [7] discussed a different generalization of these spaces. Sargent [22] dealt with the spaces $N_{\phi}(R)$ when $\mu$ is a discrete measure. Later Steigerwalt and White [24] extended Sargent's work when $\mu$ is a positive measure with the finite subset property.

The results in [12], [22], [24] are concerned with the definition of these spaces, and few fundamental properties such as separability, and reflexivity of these spaces. In [12] Lorentz has given an interesting application to Fourier analysis. Hunt [9] and O'Neil [18] have extensively applied these spaces in discussing interpolation theorems and convolution operators. For further applications in this direction, see a recent paper of Lorentz and Shimogaki [15]. Semenov [23] obtained certain embedding theorems by using Lorentz space techniques, for symmetric Banach function spaces. In spite of such wide applications of these spaces no attempt at a systematic investigation of the geometric and related properties of these spaces has yet been made. The results in the paper summarize part of the work accomplished in this direction.

The plan of the paper is as follows. After recapitulating basic definitions and properties of some of the relevant fundamental concepts such as concave functions and decreasing rearrangements in $\S \S 2,3,4$ the main results are presented in $\S \S 5,6,7$ and 8 . In $\S 5$, the Banach spaces $N_{\phi}(E)$ are introduced and their duals are identified. It is also shown that the spaces $N_{\phi}(R)$, more generally $N_{\phi}(E)$, are nonreflexive in all nontrivial cases, thus generalizing a result in [12] dealt with for the case $\phi(t)=t^{\alpha}, 0<\alpha<1$. In [6] a complete characterization of the extreme points of $U\left(N_{\phi}(R)\right)$ is provided. In [7] this result is generalized to obtain the extreme points of $U\left(N_{\phi}(E)\right)$ when $E$ is separable.

2. Basic definitions and preliminary results. All vector spaces considered here are over the real field. The real number system will be denoted by $R$, the extended real number system by $\bar{R}$, their positive parts by $R^{+}, \bar{R}^{+}$respectively. If $E$ is a normed linear space $E^{*}$ is the dual of $E$. The set $U(E)$ is the unit cell of $E$, and $S(E)$ is the boundary of $U(E)$. The norms of various Banach spaces under discussion are denoted by the same symbol \|\| unless otherwise specified. If $C$ is a convex set, the set of extreme points of $C$ is denoted by Ext $C$.

Throughout the paper $(X, \Sigma, \mu)$ is a measure space, where $\mu: \Sigma \rightarrow \bar{R}^{+}$is a countably additive measure. For measure theoretic terminology, not defined here: see Dunford and Schwartz [5], and Halmos [6]. The set of measurable functions 
with values in $R(\bar{R})$ will be denoted by $M_{R}\left(M_{\bar{R}}\right)$.

Let $E$ be a Banach space. Then $f: X \rightarrow E$ is $\mu$-measurable if

(i) $f^{-1}(G) \in \Sigma$ for all open subsets $G$ of $E$, and

(ii) for each $A \in \Sigma$ such that $\mu(A)<\infty$, there exists a set $N \in \Sigma$ with $\mu(N)=$ 0 such that the range of $f$ restricted to $A \sim N$ is in a separable subspace of $E$. The set of all $\mu$-measurable $E$-valued functions will be denoted by $M_{E}$. If $f \in M_{E}$, then $\widetilde{f}: X \rightarrow R$ is defined by $\widetilde{f}(x)=\|f(x)\|$ for all $x \in X$. It is verified that $\tilde{f} \in M_{R}$. If $A \in \Sigma$, the characteristic function of $A$ is denoted by $\chi_{A}$.

Let $X$ be a set, $S$ be a ring of subsets of $X$, and $m: S \rightarrow E$ be a function. The variation of $m$, denoted by $\bar{m}$ is defined on all subsets of $X$, by the formula $\bar{m}(A)=\operatorname{Sup} \Sigma\left\|m\left(A_{i}\right)\right\|$ where the supremum is taken over all finite families $\left\{A_{i}\right\}_{i=1}^{n}$ of pairwise disjoint sets $\left\{A_{i}\right\}_{i=1}^{n}$ of $S$ contained in $A$.

For the basic concepts of vector valued measures, see Dinculeanu [4]. Here another useful property of vector valued measures is recalled, as this has not been dealt with in [4].

A Banach space $E$ has the Radon-Nikodým property if, given any finite measure space $(X, \Sigma, \mu)$, and an $E$-valued vector measure $m$ such that

(i) $\bar{m}(X)<\infty$, and

(ii) $m \ll \mu$, i.e., $m(A)=0$ whenever $\mu(A)=0$, then there exists an $E$-valued Bochner integrable function $f$ on $X$ such that

$$
m(A)=\int_{A} f d \mu \text { for all } A \in \Sigma .
$$

For a characterization of a measure taking values in $E$ admitting a Radon-Nikodým derivative with respect to a real valued measure, see Rieffel [20]. It is known that if $E$ is reflexive then $E$ has the Radon-Nikodým property, Troyanski [29], Leonard and Sundaresan [11]. For more about the R-N property, see Phelps [19]. If $f \in M_{E}, \lambda_{f}: R^{+} \rightarrow \bar{R}^{+}$is defined by setting $\lambda_{f}(y)=\mu\{x \in X:\|f(x)\|>$ $y$ \}. This function will be used here extensively. Certain results concerning vector valued functions are required, and there seems to be no convenient reference in the literature. These results are stated here for completeness.

A sequence $\left\{f_{n}\right\}_{n \geqslant 1} \subset M_{E}$ is said to be essentially uniformly Cauchy on a set $A \in \Sigma$, if for $\epsilon>0$ there is an integer $N(\epsilon)$ such that $\left\|f_{n}(x)-f_{m}(x)\right\|<\epsilon$ if $n, m>N(\epsilon)$ for $x$-a.e. in $A$. It is almost uniformly Cauchy if for $\epsilon>0$ there exists a set $F \in \Sigma, \mu(F)<\epsilon$ such that the sequence $\left\{f_{n}\right\}_{n \geqslant 1}$ is essentially uniformly Cauchy on $X \sim F$.

Lemma 1. Let $\left\{f_{n}\right\}_{n \geqslant 1} \subset M_{E}$ be such that for all $y>0, \lambda_{f_{n}-f_{m}}(y) \rightarrow 0$ as $m, n \rightarrow \infty$. Then there is a subsequence $\left\{f_{n_{k}}\right\}_{k \geqslant 1}$ which is almost uniformly Cauchy. 
LEMma 2. Let $\left\{f_{n}\right\}_{n \geqslant 1}$ be as in Lemma 1. Then there is a function $f \in$ $M_{E}$ such that $\lambda_{f_{n}-f}(y) \rightarrow 0$ as $n \rightarrow \infty$ for all $y>0$.

The proof of Lemma 1 is very similar to Theorem D, p. 93 in [6].

Lemma 2 is a consequence of Lemma 1, and Egorov's theorem for vector valued measurable functions $[4$, p. 94 , Theorem 1$]$.

3. Concave functions, and related function spaces. Let $C$ be the set of all concave functions $\phi: \bar{R}^{+} \rightarrow \bar{R}^{+}, \phi$ nondecreasing and satisfying $\phi(0)=0=\phi(0+)$. For a discussion of such functions see Hardy, Littlewood, and Pólya [8], Stein and Weiss [25]. In particular such a function $\phi$ is locally absolutely continuous so that the derivative $\phi^{\prime}$ exists a.e. and $\phi(t)=\int_{0}^{t} \phi^{\prime}(\xi) d \xi$. In the following proposition basic properties of $\phi$ are stated.

Proposition 1. Let $\phi \in C$.

(1) If $K \in[0,1]$, then $\phi(K x) \geqslant K \phi(x)$.

(2) $\phi(x) / x$ is nonincreasing as $x$ increases.

(3) If for some $K>0$ there exist $x_{1}, x_{2}, 0<x_{1}<x_{2}$, such that $\phi\left(x_{1}\right)=$ $K x_{1}$ and $\phi\left(x_{2}\right)=K x_{2}$, then $\phi(x)=K x$ for $x \in\left[0, x_{2}\right]$.

(4) If for some $K>0$ there exist $x_{1}, x_{2}, 0<x_{1}<x_{2}$, such that $\phi\left(x_{1}\right)=$ $K=\phi\left(x_{2}\right)$, then $\phi(x)=K$ for $x \geqslant x_{2}$.

(5) If for all $x>K>0, \phi$ is a constant on some subinterval of $[0, x]$, then $\phi(x)=\phi(K)$, for all $x \geqslant K$.

(6) If there exist $x_{1}, x_{2}>0$ such that $\phi\left(x_{1}+x_{2}\right)=\phi\left(x_{1}\right)+\phi\left(x_{2}\right)$, then $\phi(x)=K x$ for some $K>0$, for all $x \in\left[0, x_{1}+x_{2}\right]$.

(7) $\phi^{\prime}(x)$ is nonnegative and nonincreasing as $x$ increases.

(8) $\phi\left(x_{1}+x_{2}\right) \leqslant \phi\left(x_{1}\right)+\phi\left(x_{2}\right)$ for all $x_{1}, x_{2} \geqslant 0$.

The proposition is a direct consequence of concavity, and continuity of $\phi$.

Let $(X, \Sigma, \mu)$ be a $\sigma$-finite measure space, and $\phi \in C$. For $f \in M_{\bar{R}}$, define $\|f\|_{1}=\int_{0}^{\infty} \phi\left(\lambda_{f}(y)\right) d y$. If $N_{\phi}(R)=\left\{f\|\| f \|_{1}<\infty\right\}$ then, identifying functions which agree $\mu$-a.e., $\left(N_{\phi}(R),\|\|_{1}\right)$ is a Banach space under the usual pointwise linear operations [24].

Let $M_{\phi}(R)$ be the set of all functions $f \in M_{\bar{R}}$ such that

$$
\|f\|_{2}=\operatorname{Sup}_{0<\phi(\mu(A))<\infty}\left\{\frac{1}{\phi(\mu(A))} \int_{A}|f| d \mu\right\}<\infty .
$$

After identifying functions which agree $\mu$-a.e. $\left(M_{\phi}(R),\|\|_{2}\right)$ is a Banach space, and if either $\mu(X)<\infty$, or $\mu$ is $\sigma$-finite and $\phi$ is unbounded, then $M_{\phi}(R)$ is isometrically isomorphic with $N_{\phi}^{*}(R)$ [24].

The norms of $N_{\phi}(R)\left(M_{\phi}(R)\right)$ are denoted either by the symbols \|\|$_{N_{\phi}(R)}$ ( \|\|$\left._{M_{\phi}(R)}\right)$ or simply by \|\|$_{1}\left(\|\|_{2}\right)$. 
The vector valued analogues of the Banach spaces $N_{\phi}(R)$ and $M_{\phi}(R)$ are introduced in $\S 5$. These are certain vector subspaces of equivalence classes of $E$ valued $\Sigma$-measurable functions.

4. The decreasing rearrangement function. The following material concerning the decreasing rearrangement function is required in the study of extreme points of $U\left(M_{\phi}(R)\right)$. For a complete discussion of this function and related results, see Luxemburg [16].

Let $(X, \Sigma, \mu)$ be a finite measure space and $f \in M_{R}$. The decreasing rearrangement function of $f, \delta_{f}$, is defined by

$$
\delta_{f}(t)=\inf \{u: \mu\{x: f(x)>u\} \leqslant t\}
$$

for $0 \leqslant t \leqslant \mu(X)$. If $f, g \in M_{\bar{R}}$, then $f$ and $g$ are equimeasurable, denoted $f \sim g$, if $\delta_{f}=\delta_{g}$.

The next proposition will be extensively used and is stated here for convenience of reference without proof. A proof may be found in [16, pp. 88, 93].

Proposition 2. Let $(X, \Sigma, \mu)$ be a finite measure space and $f_{1}, f_{2} \in M_{\bar{R}}$, then

(1) $f_{1} \sim \delta_{f_{1}}$.

(2) If $f_{1} \sim f_{2}$, then $\left|f_{1}\right| \sim\left|f_{2}\right|$.

(3) If $\left|\delta_{f_{1}}\right|=g$, then $\delta_{g}=\delta_{\left|f_{1}\right|}$.

If $f, g \in L_{1}(\mu)$, then $f<g$ will mean that $\int_{X} f d \mu=\int_{X} g d \mu$ and $\int_{0}^{t} \delta_{f}(u) d u \leqslant$ $\int_{0}^{t} \delta_{g}(u) d u$ for all $0 \leqslant t \leqslant \mu(X)$. Thus $f \sim g$ if and only if $f<g$ and $g<f$, see $[16$, p. 96$]$.

A finite measure space $\mu$ is adequate [16], if

$$
\max \left\{\int_{X} f g^{\prime} d \mu: g^{\prime} \sim g\right\}=\int_{0}^{\mu(X)} \delta_{f} \delta_{g} d t .
$$

It is known that nonatomic measures are adequate [16].

The Banach spaces $N_{\phi}(R)$, and $M_{\phi}(R)$ are examples of Banach Function Spaces extensively studied by Luxemburg and Zaanen [17]. In particular these spaces share the following properties of a Banach function space $(B,\|\|)$. (a) If $0 \leqslant f_{1} \leqslant f_{2}$, pointwise, $f_{1}, f_{2} \in B$, then $\left\|f_{1}\right\| \leqslant\left\|f_{2}\right\|$. (b) If $f_{n}, f \in M_{\bar{R}}, f_{n} \uparrow f$ pointwise then $\left\|f_{n}\right\| \uparrow\|f\|$.

A Banach Function Space $(B ;\|\|)$ is rearrangement invariant if $f_{1} \in B$, $f_{1} \sim f_{2}$ implies $f_{2} \in B$. A Banach Function Space $B$ with property (b) is universally rearrangement invariant if $B \subset L_{1}(\mu)$, and $f_{2} \in B, f_{1}<f_{2}$ imply $f_{1} \in B$. The spaces $N_{\phi}(R)$ and $M_{\phi}(R)$ are examples of universally rearrangement invariant Banach Function Spaces.

The decreasing rearrangement function of $|f|$ is denoted by $f^{*}$. Thus 


$$
f^{*}(t)=\delta_{|f|}(t)=\inf \left\{y: \lambda_{f}(y) \leqslant t\right\}, \quad t \geqslant 0 .
$$

For properties of $f^{*}$, see [8] or [30]. In particular it is noted that

$$
\int_{X}|f| d \mu=\int_{0}^{\mu(X)} f^{*}(t) d t
$$

For a proof of the preceding equation, see page 112 in [30]. For some useful properties of $f^{*}$ required in subsequent discussion, refer to pages 190 and 201 in [25]. The section is concluded with the following formulae. If $f \in N_{\phi}(R)$, then $\|f\|_{N_{\phi}(R)}=\int_{0}^{\infty} \phi^{\prime}(t) f^{*}(t) d t$, and if $f \in M_{\phi}(R)$, then

$$
\|f\|_{M_{\phi}(R)}=\operatorname{Sup}_{t>0}\left\{\frac{1}{\phi(t)} \int_{0}^{t} f^{*}(u) d u\right\} .
$$

For proofs of these formulae, see [24, Theorems 2.3 and 3.3].

5. Banach spaces $N_{\phi}(E)$, and their duals. Let $(X, \Sigma, \mu)$ be a $\sigma$-finite measure space, $E$ be a Banach space, and let $\phi \in C$. For $f \in M_{E}$, define

$$
\|f\|_{N_{\phi}(E)}=\int_{0}^{\infty} \phi\left(\lambda_{f}(y)\right) d y .
$$

From the definition of $\lambda_{f}$, it is verified that $\lambda_{f}$ is nonincreasing as $y$ increases. Thus $\lambda_{f}$ is Lebesgue measurable, and since $\phi$ is absolutely continuous, $\phi \circ \lambda_{f}$ is integrable. Let $N_{\phi}(E)$ be the space of $E$-valued measurable functions $f$, such that $\|f\|_{N_{\phi}(E)}$ is finite. After identifying functions agreeing $\mu$ a.e., and defining addition and scalar multiplication pointwise, $N_{\phi}(E)$ with norm \|\|$_{N_{\phi}(E)}$ is verified to be a normed linear space after noting that for $f, g \in M_{E}$,

$$
\|f\|_{N_{\phi}(E)}=\|\tilde{f}\|_{N_{\phi}(R)}, \quad \|\left(\widetilde{f+g)}\left\|_{N_{\phi}(R)} \leqslant\right\| \widetilde{f}\left\|_{N_{\phi}(R)}+\right\| \tilde{g} \|_{N_{\phi}(R)} .\right.
$$

The proofs are direct consequences of definitions and are not supplied. For notational convenience the norm \|\|$_{N_{\phi}(E)}$ is sometimes denoted by \|\|$_{1}$.

THEOREM 1. $N_{\phi}(E)$ is a Banach space.

The proof is very similar to that of Theorem 2.1 of [24]. The necessary modifications are effected using Lemmas 1 and 2.

Before obtaining the dual of $N_{\phi}(E)$, a few useful lemmas are established.

LEMmA 3. The norm in $N_{\phi}(E)$ is absolutely continuous i.e. if $f \in N_{\phi}(E)$, and $\left\{A_{n}\right\}_{n \geqslant 1}$ is a sequence in $\Sigma$ with $\mu\left(A_{n}\right) \downarrow 0$, then $\left\|f \chi_{A_{n}}\right\|_{1} \rightarrow 0$.

Proof. From equation (1), if $f \in N_{\phi}(E)$, then $\lim _{n \rightarrow \infty} \int_{n}^{\infty} \phi\left(\lambda_{f}(y)\right) d y=0$. Let now $\left\{A_{n}\right\}_{n \geqslant 1}$ be as in the statement of the lemma. Let $\epsilon>0$. Choose $K$ such that $\int_{K}^{\infty} \phi\left(\lambda_{f}(y)\right) d y<\epsilon / 2$. Choose $N$ such that for $n \geqslant N, \phi\left(\mu\left(A_{n}\right)\right)<\epsilon / 2 K$. 
Thus if $n>N$,

$$
\begin{aligned}
\left\|f \chi_{A_{n}}\right\|_{1} & <\int_{0}^{K} \phi \mu\left\{x \in A_{n}:\|f(x)\|>y\right\} d y+\epsilon / 2 \\
& \leqslant K \phi\left(\mu\left(A_{n}\right)\right)+\epsilon / 2<\epsilon .
\end{aligned}
$$

If $(X, \Sigma, \mu)$ is a measure space let $S$ be the ring of sets in $\Sigma$ of finite $\mu$ measure. $S$ is a $\delta$-ring.

LEMMA 4. If either $\mu(X)<\infty$, or $(X, \Sigma, \mu)$ is a $\sigma$-finite measure space, and $\phi$ is unbounded then the $S$-step functions are dense in $N_{\phi}(E)$.

Proof. Let $f \in N_{\phi}(E)$. Then as a consequence of Theorem 2, p. 99 in [4], it follows that there is a sequence $f_{n}$ of $E$-valued $\Sigma$-step functions with $\left\|f_{n}(x)\right\| \leqslant$ $\|f(x)\|$ for all $n \geqslant 1$, for all $x \in X$, such that $f_{n} \rightarrow f$ pointwise $\mu$ a.e.

For $n \geqslant 1$, let $B_{n}=\left\{x \in X \mid\left\|f_{n}(x)\right\|>1 / n\right\}$. Then if $\mu$ is not finite by appealing to the inequality $\phi\left(\mu\left(B_{n}\right)\right) / n \leqslant\|f\|_{1}$, it follows that $\mu\left(B_{n}\right)<\infty$, since in this case $\phi$ is unbounded. Let $g_{n}=f_{n} \chi_{B_{n}}$. Then $g_{n}$ are $S$-step functions, $\left\|g_{n}(x)\right\| \leqslant\|f(x)\|$ for all $x \in X$ and for $n \geqslant 1$.

By Egorov's theorem, p. 94 in [4], for $m \geqslant 1$ there exist $C_{m} \in S$ such that $\mu\left(B_{m} \sim C_{m}\right)<1 / m$ and $g_{m} \rightarrow f$ uniformly on $C_{m}$. For all $m, n \geqslant 1$,

$$
\left\|g_{n}-f\right\|_{1} \leqslant\left\|\left(g_{n}-f\right) \chi_{C_{m}}\right\|_{1}+\left\|\left(g_{n}-f\right) \chi_{B_{m} \sim C_{m}}\right\|_{1}
$$

$$
+\left\|\left(g_{n}-f\right) \chi_{X \sim B_{m}}\right\|_{1} .
$$

Now given $\epsilon>0$, since $\mu\left(B_{m} \sim C_{m}\right) \rightarrow 0$ as $m \rightarrow \infty$, and \|\|$_{1}$ is absolutely continuous there exists an $m_{1}$ such that for $m>m_{1},\left\|2 f \chi_{B_{m} \sim C_{m}}\right\|_{1}<\epsilon / 3$. Thus for $m>m_{1},\left\|\left(g_{n}-f\right) \chi_{B_{m} \sim C_{m}}\right\|_{1}<\epsilon / 3$.

Since $x \in X \sim B_{m}$ implies $\|f(x)\| \leqslant 1 / m$,

$$
\begin{aligned}
\left\|\left(g_{n}-f\right) \chi_{X \sim B_{m}}\right\|_{1} & \leqslant\left\|g_{n} \chi_{X \sim B_{m}}\right\|_{1}+\left\|f \chi_{X \sim B_{m}}\right\|_{1} \\
& \leqslant\left\|2 f \chi_{X \sim B_{m}}\right\|_{1} \leqslant 2 \int_{0}^{1 / m} \phi\left(\lambda_{f}(y)\right) d y \rightarrow 0
\end{aligned}
$$

as $m \rightarrow \infty$. Hence there is an $m_{2}$ such that if $m>m_{2},\left\|\left(g_{n}-f\right) \chi_{X \sim B_{m}}\right\|_{1}<$ $\epsilon / 3$. Let $p=\operatorname{Max}\left(m_{1}, m_{2}\right)$. Since $g_{m} \rightarrow f$ uniformly on $C_{p}$, there exists $N$ such that if $n>N$ then $\left\|\left(g_{n}-f\right)(x)\right\|<\epsilon / 3 \phi\left(\mu\left(C_{p}\right)\right)$ for all $x \in C_{p}$. Hence for all $n>$ $N$,

$$
\left\|\left(g_{n}-f\right) \chi_{C_{p}}\right\|_{1} \leqslant \int_{0}^{\epsilon / 3 \phi\left(\mu\left(C_{p}\right)\right)} \phi\left(\mu\left(C_{p}\right)\right) d y=\epsilon / 3 .
$$

Thus if $n>N,\left\|\left(g_{n}-f\right)\right\|_{1}<\epsilon$. Thus $S$-step functions are dense in $N_{\phi}(E)$.

Next the dual of $N_{\phi}(E)$ will be identified with a space $M$ of vector valued measures. Let $M$ be the space of all $E^{*}$-valued measures on $\Sigma$, such that 


$$
\|m\|=\operatorname{Sup}\{\bar{m}(A) / \phi(\mu(A)): 0<\phi(\mu(A))<\infty\}<\infty
$$

where $\bar{m}$, the variation of $m$ is absolutely continuous with respect to $\mu$, i.e. $\mu(A)=0$ implies $\bar{m}(A)=0$. From Remark 10, p. 36 in [4] it is verified that $(M,\|\|)$ is a Banach space.

It will now be shown that $M$ can be identified with the dual space of $N_{\phi}(E)$.

THEOREM 2. If either $\mu(X)<\infty$ or $(X, \Sigma, \mu)$ is $\sigma$-finite and $\phi$ is unbounded, then $M$ is isometrically isomorphic to $N_{\phi}(E)^{*}$ under the mapping $\alpha: M \rightarrow N_{\phi}(E)^{*}$ where $\alpha(m) f=\int_{X} f d m$ for all $f \in N_{\phi}(E)$.

Proof. It will first be shown that for any $m \in M$ and $f \in N_{\phi}(E) f$ is $m$-integrable. It is claimed first that

$$
\bar{m}(A) \leqslant\|m\| \phi(\mu(A)) \text { for all } A \in S .
$$

If $\phi(\mu(A))=0$, then $\mu(A)=0$ so that $\bar{m}(A)=0$ since $m \in M$. If $\infty>\phi(\mu(A))>$ 0 , then $\|m\| \geqslant \bar{m}(A) / \phi(\mu(A))$ so that for all $A \in S$ the given inequality holds. If $f \in N_{\phi}(E)$,

$$
\int_{X}\|f(x)\| d \bar{m}(x)=\int_{X} \tilde{f} d \bar{m}=\int_{0}^{\infty} \bar{m}\{x \in X: \tilde{f}(x)>y\} d y
$$

where the last equality follows from equation 4.8, p. 112 of vol. II [30]. Thus

$$
\begin{aligned}
\int_{X}\|f(x)\| d \bar{m}(x) & \leqslant \int_{0}^{\infty}\|m\| \phi(\mu\{x \in X: \tilde{f}(x)>y\}) d y \\
& =\|m\| \int_{0}^{\infty} \phi\left(\lambda_{f}(y)\right) d y=\|m\|\|\widetilde{f}\|_{N_{\phi}(R)}=\|m\|\|f\|_{1},
\end{aligned}
$$

so that $\|f(\cdot)\|$ is $\bar{m}$-integrable.

By Theorem 2, p. 99 of [4], there exists a sequence $\{g\}_{n \geqslant 1}$ of $E$-valued $\Sigma$ step functions such that $g_{n} \rightarrow f$ pointwise $\mu$-a.e. Choose $\left\{A_{n}\right\}_{n \geqslant 1} \subset S$ with $A_{n} \subseteq A_{n+1}$ and such that $\bigcup_{n>1} A_{n}=X$. Defining $h_{n}=g_{n} \chi_{A_{n}}$, it follows that $h_{n} \rightarrow f$ pointwise $\mu$-a.e. and the $h_{n}$ are $S$-step functions. Since $\mu(A)=0$ implies that $m(A)=0$ it follows that $h_{n} \rightarrow f$ pointwise $m$-a.e. Thus the function $f$ is $m$-measurable.

Therefore by Proposition 4, p. 122 [4], $f$ is $m$-integrable and

$$
\left|\int_{X} f d m\right| \leqslant \int_{X} f d \bar{m} \leqslant\|m\|\|f\|_{1} .
$$

For $m \in M$ and $f \in N_{\phi}(E)$ it is thus possible to define $\alpha(m) f=\int_{X} f d m$ and $|\alpha(m) f| \leqslant\|m\|\|f\|_{1}$. Since $\alpha(m)$ is clearly linear, $\alpha(m) \in N_{\phi}(E)^{*}$ and $\|\alpha(m)\| \leqslant$ $\|m\|$.

It will now be shown that $\|S(m)\|=\|m\|$. Let $A \in \Sigma$ with $0<\phi(\mu(A))<$ $\infty$ and choose $\epsilon>0$. By the definition of $\bar{m}$, there exist $\left\{A_{i}\right\}_{i=1}^{n},\left\{A_{i}\right\}$ pairwise disjoint subsets of $A, A_{i}$ in $\Sigma$ such that 


$$
\bar{m}(A) \leqslant \sum_{i=1}^{n}\left\|m\left(A_{i}\right)\right\|+\frac{\epsilon \phi(\mu(A))}{2} .
$$

For each $A_{i}$ there exists $x_{i} \in E$ with $\left\|x_{i}\right\|=1$ such that

$$
\left\|m\left(A_{i}\right)\right\| \leqslant m\left(A_{i}\right) x_{i}+\epsilon \phi(\mu(A)) / 2 n .
$$

Let $f=\sum_{i=1}^{n} x_{i} \chi_{A_{i}} / \phi(\mu(A))$. Then $\|f\|_{1}=\phi\left(\mu\left(\sum_{i=1}^{n} A_{i}\right)\right) / \phi(\mu(A)) \leqslant 1$. Thus

$$
\begin{aligned}
\|\alpha(m)\| & \geqslant|\alpha(m) f|=\left|\int_{X} f d m\right| \\
& \geqslant \frac{\sum_{i=1}^{n}\left(\left\|m\left(A_{i}\right)\right\|-\epsilon \phi(\mu(A)) / 2 n\right)}{\phi(\mu(A))} \\
& \geqslant \frac{(\bar{m}(A)-\epsilon \phi(\mu(A)) / 2)}{\phi(\mu(A))}-\frac{\epsilon}{2}=\frac{\bar{m}(A)}{\phi(\mu(A))}-\epsilon .
\end{aligned}
$$

Therefore $\|\alpha(m)\| \geqslant \bar{m}(A) / \phi(\mu(A))$ if $0<\phi(\mu(A))<\infty$, so that $\|\alpha(m)\| \geqslant\|m\|$. It therefore follows that $\|S(m)\|=\|m\|$. It is seen from the definition that $S$ is linear and thus $\alpha$ is a linear isometry from $M$ into $N_{\phi}(E)^{*}$. It remains to be shown that $\alpha$ is onto $N_{\phi}(E)^{*}$.

Let $l \in N_{\phi}(E)^{*}$. Define $m: \alpha \rightarrow E^{*}$ by $m(A) x=l\left(x \chi_{A}\right)$ if $A \in S$ and $x \in$ $E$. Then for $x, y \in E$ and scalars $\alpha, \beta$,

$$
m(A)(\alpha x+\beta y)=l\left([\alpha x+\beta y] \chi_{A}\right)=l\left(\alpha x \chi_{A}+\beta_{y} \chi_{A}\right)=\alpha m(A) x+\beta m(A) y .
$$

Also

$$
\|m(A)\|=\sup _{\|x\|<1}\left|l\left(x \chi_{A}\right)\right| \leqslant \sup _{\|x\|<1}\|l\|\left\|x \chi_{A}\right\|_{N_{\phi}(E)}=\|l\| \phi(\mu(A))<\infty .
$$

Thus $m(A) \in E^{*}$ if $A \in S$.

It will next be shown that $m$ is a measure. It is seen that $m(\varnothing)=0$ and $m(A \cup B)=m(A)+m(B)$ if $A \cap B=\varnothing$ so that $m$ is a finitely additive set function. Let $\left\{A_{i}\right\}_{i \geqslant 1}$ be a pairwise disjoint family of elements of $S$ such that $A=$ $\bigcup_{i>1} A_{i} \in S$. If $\mu(X)<\infty$, then also $\mu(A)<\infty$. If $\mu(X)=\infty$, then $\phi$ is unbounded and therefore $A \in S$ implies $\mu(A)<\infty$. Thus in either case $\mu(A)<\infty$ so that $\mu\left(\bigcup_{i=m}^{\infty} A_{i}\right) \rightarrow 0$ as $m \rightarrow \infty$, since $\mu$ is countably additive. Thus

$$
\begin{aligned}
\left\|m(A)-\sum_{i=1}^{n} m\left(A_{i}\right)\right\|_{E^{*}} & =\left\|m(A)-m\left(\bigcup_{i=1}^{n} A_{i}\right)\right\|_{E^{*}}=\left\|m\left(A-\bigcup_{i=1}^{n} A_{i}\right)\right\|_{E^{*}} \\
& =\left\|m\left(\bigcup_{i=n+1}^{\infty} A_{i}\right)\right\|_{E^{*}} \leqslant\|l\| \phi\left(\mu\left(\bigcup_{i=n+1}^{\infty} A_{i}\right)\right) \rightarrow 0
\end{aligned}
$$


since $\mu\left(\bigcup_{i=n+1}^{\infty} A_{i}\right) \rightarrow 0$ as $n \rightarrow \infty$. Hence $m\left(\bigcup_{i \geqslant 1} A_{i}\right)=\Sigma_{i>1} m\left(A_{i}\right)$ and $m$ is therefore a measure on the $\delta$-ring $S$ into $E^{*}$.

Next it will be shown that $m \in M$. Let $A \in S$ with $\mu(A)>0$. Choose $\left\{A_{i}\right\}_{i=1}^{n} \subset S$ pairwise disjoint subsets of $A$ and let $\epsilon>0$ be given. For each $i$, $1 \leqslant i \leqslant n$, there exists $x_{i} \in E$ with $\left\|x_{i}\right\|=1$ such that

$$
m\left(A_{i}\right) x_{i} \geqslant\left\|m\left(A_{i}\right)\right\|-\epsilon / n .
$$

Hence $\Sigma_{i=1}^{n}\left\|m\left(A_{i}\right)\right\| \leqslant \sum_{i=1}^{n} m\left(A_{i}\right) x_{i}+\epsilon$. However

$$
\sum_{i=1}^{n} m\left(A_{i}\right) x_{i}=\sum_{i=1}^{n} l\left(x_{i} \chi_{A_{i}}\right) \leqslant\|l\|\left\|\sum_{i=1}^{n} x_{i} x_{A_{i}}\right\|_{1}=\|l\| \phi\left(\mu\left(\bigcup_{i=1}^{n} A_{i}\right)\right) .
$$

Consequently

$$
\sum_{i=1}^{n}\left\|m\left(A_{i}\right)\right\| \leqslant\|l\| \phi\left(\mu\left(\bigcup_{i=1}^{n} A_{i}\right)\right)+\epsilon \leqslant\|l\| \phi(\mu(A))+\epsilon .
$$

Hence $\Sigma_{i=1}^{n}\left\|m\left(A_{i}\right)\right\| \leqslant\|l\| \phi(\mu(A))$. Therefore $\bar{m}(A) \leqslant\|l\| \phi(\mu(A))$ so that $\|m\| \leqslant$ $\|l\|<\infty$. It is verified appealing to definitions that $m \ll \mu$. Thus $m \in M$.

It will now be shown that $\alpha(m)=l$ thus proving that $\alpha$ is onto $N_{\phi}(E)^{*}$. Assume first that $f \in N_{\phi}(E)$ is an $S$-step function. Therefore $f=\sum_{i=1}^{n} x_{i} \chi_{A_{i}}$ with $x_{i} \in E$ and $A_{i} \in S$. Then by direct computation

$$
\alpha(m) f=\sum_{i=1}^{n} \int_{A_{i}} x_{i} d m=\sum_{i=1}^{n} m\left(A_{i}\right) x_{i}=l\left(\sum_{i=1}^{n} x_{i} \chi_{A_{i}}\right)=l(f) .
$$

Thus $\alpha(m)(f)=l(f)$ for all $E$-valued $S$-step functions $f$. Next let $g \in N_{\phi}(E)$ be arbitrary. By Lemma 4 there exists a sequence $\left\{f_{n}\right\}_{n>1}$ of $E$-valued $S$-step functions such that $f_{n} \rightarrow g$ in $N_{\phi}(E)$. Then, since both $\alpha(m)$ and $l$ are elements of $N_{\phi}(E)^{*}$,

$$
\alpha(m)(g)=S(m)\left(\lim _{n \rightarrow \infty} f_{n}\right)=\lim _{n \rightarrow \infty} l\left(f_{n}\right)=l(g) .
$$

Thus $\alpha(m)=l$ so that $S$ is onto $N_{\phi}(E)^{*}$ and $\alpha$ is therefore an isometric isomorphism from $M$ onto $N_{\phi}(E)^{*}$.

REMARK. It should be noted that the conclusion of Theorem 2 may be false, even for $N_{\phi}(R)$, in the case when $(X, \Sigma, \mu)$ is a $\sigma$-finite measure space but $\phi$ is a bounded function. For example, let $X=R^{+}$and $\mu$ be the Lebesgue measure. If $A \in \Sigma$, then $\left\|\chi_{A}\right\|_{1}=\phi(\mu(A))<\infty$ since $\phi$ is bounded, so that $\chi_{A} \in$ $N_{\phi}(R)$. If $L \in N_{\phi}(R)^{*}$, then $m: \Sigma \rightarrow R$ is to be a measure such that $L(f)=$ $\int_{X} f d m$ for all $f \in N_{\phi}(R)$. Thus, in particular, $L\left(\chi_{A}\right)=\int_{X} \chi_{A} d m=m(A)$ for all $A \in \Sigma$. Let $A_{n}=[n, n+1)$ for $n \geqslant 0$. Then $\bigcup_{n \geq 0} A_{n}=R^{+}$and the $A_{n}$ are pairwise disjoint. Hence, since $m$ is countably additive,

$$
L\left(\chi_{R^{+}}\right)=m\left(R^{+}\right)=m\left(\bigcup_{n \geqslant 0} A_{n}\right)=\sum_{n \geqslant 0} m\left(A_{n}\right)=\sum_{n \geq 0} L\left(\chi_{[n, n+1)}\right) .
$$


It will be shown, however, that there exists an $L \in N_{\phi}(R)^{*}$ such that

$$
L\left(\chi_{R^{+}}\right) \neq \sum_{n \geqslant 0} L\left(\chi_{[n, n+1)}\right)
$$

so that this $L$ cannot be represented by a measure $m$ in the above manner.

For $f \in N_{\phi}(R)$, ess $\lim _{t \rightarrow \infty} f(t)=\alpha$ will mean that there exists $N \in \Sigma$ with $\mu(N)=0$ such that $\lim _{t \rightarrow \infty ; t \notin N} f(t)=\alpha$. Let $U=\left\{f \in N_{\phi}(R): \operatorname{ess} \lim _{t \rightarrow \infty} f(t)\right.$ exists\}. Then $U \neq \varnothing$ and $U$ is a subspace of $N_{\phi}(R)$. For $f \in U$, define

$$
l(f)=\underset{t \rightarrow \infty}{\operatorname{ess} \lim } f(t)
$$

so that $l$ is a linear functional on $U$. It will be shown that $l$ is continuous. Suppose $f \in U$ is such that $l(f)=\alpha>0$. Thus there exists $N \in \Sigma$ with $\mu(N)=0$ so that $\lim _{t \rightarrow \infty ; t \notin N} f(t)=\alpha$.

Choose $\epsilon>0$ and such that $\epsilon<\alpha$. Then there exists $T>0$ so that $t>T$ and $t \notin N$ implies that $|f(t)-\alpha|<\epsilon$. Thus

$$
\left\{t \in R^{+}:|f(t)|>\alpha-\epsilon\right\} \supseteq\left\{t \in R^{+} \sim N: t>T\right\} .
$$

Therefore $\lambda_{f}(\alpha-\epsilon) \geqslant \mu\left\{t \in R^{+} \sim N: t>T\right\}=\infty$. Hence

$$
\begin{aligned}
\|f\|_{N_{\phi}(R)} & =\int_{0}^{\infty} \phi\left(\lambda_{f}(y)\right) d y \geqslant \int_{0}^{\alpha-\epsilon} \phi\left(\lambda_{f}(y)\right) d y \\
& \geqslant \int_{0}^{\alpha-\epsilon} \phi\left(\lambda_{f}(\alpha-\epsilon)\right) d y=(\alpha-\epsilon) \phi(\infty) .
\end{aligned}
$$

Since this is true for all $\epsilon$ such that $0<\epsilon<\alpha$, it follows that $\|f\|_{1} \geqslant \alpha \phi(\infty)$. Thus $l(f) \leqslant\|f\|_{1} / \phi(\infty)$ whenever $l(f)>0$. Therefore $|l(f)| \leqslant\|f\|_{N_{\phi}(R)} / \phi(\infty)$ for all $f \in U$. Hence $l \in U^{*}$. Now the Hahn-Banach theorem assures the existence of an extension $L$ of $l$ to all of $N_{\phi}(R)$ with $\|L\|=\|l\|$. Then $L \in N_{\phi}(R)^{*}$. However

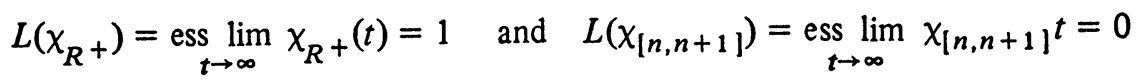

so that

$$
L\left(\chi_{R^{+}}\right) \neq \sum_{n \geq 0} L\left(\chi_{[n, n+1)}\right) .
$$

Thus $L$ cannot be represented by a measure $m$ in the above manner.

Let $F$ be a Banach space. The vector analogue of the space $M_{\phi}(R)$ is defined as follows. For $f \in M_{F}$ let $\|f\|_{M_{\phi}(F)}$, denoted some times by $\|f\|_{2}$ when $F$ is understood, be defined by

$$
\|f\|_{2}=\sup _{A \in \Sigma ; 0<\phi(\mu(A))<\infty}\left\{\frac{1}{\phi(\mu(A))} \int_{A}\|f(x)\| d \mu(x)\right\} .
$$

Let $M_{\phi}(F)=\left\{f: f \in M_{F},\|f\|_{2}<\infty\right\}$. After identifying functions agreeing $\mu$ a.e. $\left(M_{\phi}(F),\|\|_{2}\right)$ is a Banach space. The verification of this is very similar to that of Theorem 3.1 in [24]. 
If $E^{*}$ has the Radon-Nikodým property then $N_{\phi}(E)^{*}$ could be identified with $M_{\phi}\left(E^{*}\right)$, and this is the content of the next theorem. It might be recalled that $E^{*}$ has the Radon-Nikodým property if $E$ is isomorphic to a Banach space with a Fréchet differentiable norm [11], in particular if $E$ is reflexive [29]. More general results are known, and for this see a recent paper of Phelps [19], and the list of references therein.

THEOREM 2A. Let $E$ be a Banach space such that $E^{*}$ has the Radon-Nikodym property. If either $\mu(X)<\infty$ or $(X, \Sigma, \mu)$ is $\sigma$-finite and $\phi$ is unbounded, then $N_{\phi}(E)^{*}$ is isometrically isomorphic with $M_{\phi}\left(E^{*}\right)$ under the mapping $L$ : $N_{\phi}(E)^{*} \rightarrow M_{\phi}\left(E^{*}\right)$ where $L(l)=g$ and $g$ is such that

$$
l(f)=\int_{X} g(x) f(x) d \mu(x) \text { for all } f \in N_{\phi}(E) .
$$

PROOF. It will first be shown that $M$ and $M_{\phi}\left(E^{*}\right)$ are isometrically isomorphic under the mapping $T: M \rightarrow M_{\phi}\left(E^{*}\right)$ where $T(m)=g$ and $g$ is such that $m(A)=\int_{A} g d \mu$ for all $A \in S$.

Choose $m \in M$ and $\left\{A_{n}\right\}_{n \geqslant 1} \subset S$ such that $X=\bigcup_{n \geqslant 1} A_{n}$ and the sets $A_{n}$ are pairwise disjoint. Then, for each $n,\left(A_{n},\left.\Sigma\right|_{A_{n}},\left.\mu\right|_{A_{n}}\right)$ is a finite measure space. As is shown in the proof of Theorem $2, \bar{m}(A) \leqslant\|m\| \phi(\mu(A))$ for all $A \in$ $S$. Thus, since $\mu\left(A_{n}\right)<\infty$, it follows that $\bar{m}\left(A_{n}\right)<\infty$. Also, if $\mu(A)=0$, then $\bar{m}(A)=0$ so that $m(A)=0$ and therefore $\left.\left.m\right|_{A_{n}} \ll \mu\right|_{A_{n}}$. Consequently, since $E^{*}$ has the Radon-Nikodým property, there exists a unique $E^{*}$-valued Bochner integrable function $g_{n}$ on $A_{n}$ such that $m(A)=\int_{A} g_{n} d \mu$ for all $A \in S$ with $A \subset$ $A_{n}$. It may be assumed that $g_{n}$ is defined on all of $X$ and that $g \mid X \sim A_{n}=0$.

Define $g: X \rightarrow E^{*}$ by $g(x)=\Sigma_{n \geqslant 1} g_{n}(x)$ for all $x \in X$. Then $\|g(x)\|=$ $\Sigma_{n \geqslant 1}\left\|g_{n}(x)\right\|$ for all $x \in X$ since the functions $g_{n}$ have disjoint support. The function $g$ will be measurable since each $g_{n}$ is measurable. Further, if $A \in S$, then it is verified that $\bar{m}(A)=\int_{A}\|g(x)\| d \mu(x)$. Thus $\|g(\cdot)\|$ is $\mu$-integrable on $A$, and therefore $g$ is $\mu$-integrable on $A$. Hence, for all $A \in S$,

$$
m(A)=\int_{A} g d \mu \text { and } \bar{m}(A)=\int_{A}\|g(x)\| d \mu(x) .
$$

Next, it will be shown that $g \in M_{\phi}\left(E^{*}\right)$.

$$
\begin{aligned}
\|g\|_{M_{\phi}\left(E^{*}\right)} & =\sup _{A \in S ; \mu(A)>0}\left\{\frac{1}{\phi(\mu(A))} \int_{A}\|g(x)\| d \mu(x)\right\} \\
& =\sup _{A \in S ; \mu(A)>0}\left\{\frac{\bar{m}(A)}{\phi(\mu(A))}\right\}=\|m\|<\infty .
\end{aligned}
$$

Consequently $g \in M_{\phi}\left(E^{*}\right)$, and if $T: M \rightarrow M_{\phi}\left(E^{*}\right)$ is defined as above, then $T$ is well defined and

$$
\|T(m)\|_{M_{\phi}\left(E^{*}\right)}=\|g\|_{M_{\phi}\left(E^{*}\right)}=\|m\| .
$$


Further by Corollary 1, p. 187 in [4] it follows that $T$ is a linear isometry. To show that $T$ is onto, let $g \in M_{\phi}\left(E^{*}\right)$. Then since $\|g\|_{M_{\phi}\left(E^{*}\right)}<\infty$, it follows that $\int_{A}\|g(x)\| d \mu(x)<\infty$ for all $A \in S$ so that $g$ is locally $\mu$-integrable. Thus if $m$ : $S \rightarrow E^{*}$ is defined by

$$
m(A)=\int_{A} g d \mu \text { for all } A \in S,
$$

then $m$ is a measure and,

$$
\begin{aligned}
\|m\| & =\sup _{A \in S ; \mu(A)>0}\left\{\frac{\bar{m}(A)}{\phi(\mu(A))}\right\} \\
& =\sup _{A \in S ; \mu(A)>0}\left\{\frac{1}{\phi(\mu(A))} \int_{A}\|g(x)\| d \mu(x)\right\}=\|g\|_{M_{\phi}\left(E^{*}\right)^{\circ}}
\end{aligned}
$$

Thus $m \in M$ and, since $T(m)=g$, it follows that $T$ is onto, and therefore $T$ is an isometric isomorphism.

It follows from what has just been proven together with Theorem 2 that $L=T \circ S^{-1}: N_{\phi}(E)^{*} \rightarrow M_{\phi}\left(E^{*}\right)$ is an isometric isomorphism. Here, if $l \in$ $N_{\phi}(E)^{*}$, then $L(l)=g \in M_{\phi}\left(E^{*}\right)$ where $l(f)=\int_{X} f d m$ for all $f \in N_{\phi}(E)$. Since $m(A)=\int_{A} g d \mu$ it is verified that $l(f)=\int_{X} g(x) f(x) d \mu(x)$ for all $f \in N_{\phi}(E)$, using Proposition 4, p. 122, and Proposition 13, p. 136 in [4], completing the proof of the theorem.

It will be proven next that if $(X, \Sigma, \mu)$ is a nonatomic measure space and $E$ is a Banach space, then $N_{\phi}(E)$ is not reflexive. This generalizes a theorem of Lorentz [12], stating that $N_{\phi}(R)$ is not reflexive if $\phi(x)=x^{\alpha}$ for some $\alpha, 0<$ $\alpha<1$, and the underlying measure space is $(0,1)$ with the Lebesgue measure. The proof presented below uses altogether different techniques.

THEOREM 3. If $(X, \Sigma, \mu)$ is a nonatomic measure space and $\mu(X)<\infty$ or $(X, \Sigma, \mu)$ is $\sigma$-finite and $\phi$ is unbounded and $E$ is a Banach space, then $N_{\phi}(E)$ is not reflexive.

Since $N_{\phi}(R)$ is canonically imbedded in $N_{\phi}(E)$, it is enough to show that $N_{\phi}(R)$ is not reflexive.

In the remainder of this proof, $\nu$ will be used to denote Lebesgue measure on the real line $R$.

Let $g(t)=(1-t) \phi^{\prime}(t)-\phi(t)$ for $t \in[0, \infty)$. Then, since $\phi(t) \downarrow 0$ as $t \rightarrow$ $0^{+}$and $(1-t) \phi^{\prime}(t)$ increases as $t \rightarrow 0^{+}$, there exists an $a, 0<a<1$, such that $g(t) \geqslant 0$ for $t \in[0, a] v$-a.e. Choose $a$ so that $a<\mu(X)$. Also, $g(t)$ is a nonincreasing function for $t \in[0, a] \nu$-a.e. Define

$$
h(t)= \begin{cases}g(t), & t \in[0, a], \\ 0, & t \in(a, \infty) .\end{cases}
$$


Then $h(t) \geqslant 0$ and nonincreasing for $t \in[0, \infty) v$-a.e. Let

$$
f(t)= \begin{cases}(1-t) \phi(t), & t \in[0, a], \\ (1-a) \phi(a), & t \in(a, \infty) .\end{cases}
$$

Since $\phi$ is locally absolutely continuous, it follows that $f$ is locally absolutely continuous. But $f^{\prime}(t)=h(t)$ for $t v$-a.e., so that

$$
f(t)=\int_{0}^{t} h(y) d y+f(0)=\int_{0}^{t} h(y) d y,
$$

for $t v$-a.e. Since $h: R^{+} \rightarrow R^{+}$is Lebesgue measurable, there exists $\left\{s_{n}\right\}_{n \geqslant 1}$ an increasing sequence of nonnegative step functions such that $s_{n} \rightarrow h$ pointwise $\nu$ a.e. (see Theorem 8, p. 85 in [6]).

It is next claimed that there exists $\left\{P_{n}\right\}_{n \geqslant 1}$, with $P_{n}: X \rightarrow R^{+}$, a sequence of increasing $\Sigma$-step functions such that $P_{n}^{*}=s_{n}^{*}$ for all $n \geqslant 1$. If $s_{1}=\sum_{i=1}^{n} c_{i} \chi_{E_{i}}$ with $c_{i}>0, E_{i}$ pairwise disjoint Lebesgue measurable sets, and, since support $h \subset[0, a), \nu\left(\bigcup_{i=1}^{n} E_{i}\right) \leqslant a<\mu(X)$, then, since $(X, \Sigma, \mu)$ is nonatomic, it is possible to choose $D_{i} \in \Sigma, D_{i}$ pairwise disjoint and such that $\mu\left(D_{i}\right)=\nu\left(E_{i}\right)$ for $1 \leqslant$ $i \leqslant n$. If $P_{1}=\sum_{i=1}^{n} c_{i} \chi_{D_{i}}$ then $P_{1}$ is a nonnegative $\Sigma$-step function and clearly $P_{1}^{*}=s_{1}^{*} . s_{2}$ is defined by subdividing the sets $E_{i}$ and giving $s_{2}$ new values on these subdivisions and, if necessary, adding other sets $E_{i}^{\prime}$ which can be assumed to be disjoint from $\bigcup_{i=1}^{n} E_{i}$. Again since $(X, \Sigma, \mu)$ is nonatomic, it is possible to subdivide the sets $D_{i}$ in the same fashion as the $E_{i}$ are subdivided and add additional sets $D_{i}^{\prime}$, disjoint from $\bigcup_{i=1}^{n} D_{i}$, and with $\mu\left(D_{i}^{\prime}\right)=\nu\left(E_{i}^{\prime}\right)$. If $P_{2}$ is given the same values as $s_{2}$ (on these sets of equal measure), then $P_{2}^{*}=s_{2}^{*}$ and $P_{2} \geqslant P_{1}$ since $s_{2} \geqslant s_{1}$. All the functions $P_{n}$ may be constructed in a similar fashion.

Let $P(x)=\lim _{n \rightarrow \infty} P_{n}(x)$ for all $x \in X$. This limit exists since the $P_{n}$ are increasing functions. Then $P$ is a $\mu$-measurable function, Theorem A, p. 84 in [6].

Since $s_{n}(t) \leqslant s_{n+1}(t)$ for all $t \geqslant 0$ and $h(t)=\lim _{n \rightarrow \infty} s_{n}(t)$, it follows from Lemma 3.5 in [25] that, for each $t>0, s_{n}^{*}(t)$ increases monotonically to $h^{*}(t)$ as $n \rightarrow \infty$. Similarly, since $P_{n}(x) \leqslant P_{n+1}(x)$ for all $x \in X$ and $P(x)=\lim _{n \rightarrow \infty} P_{n}(x)$, it follows from the same proposition that $P_{n}^{*}(t)$ increases monotonically to $P^{*}(t)$ for each $t>0$. But $P_{n}^{*}=s_{n}^{*}$ so that $P^{*}(t)=h^{*}(t)$ for all $t>0$. Since $h(t) \geqslant 0$ and $h$ is nonincreasing it follows that $h^{*}(t)=h(t)$ and consequently $P^{*}(t)=h(t)$ for all $t>0$.

Since $(X, \Sigma, \mu)$ is $\sigma$-finite, by (B) in $\S 4$,

$$
\begin{aligned}
\|P\|_{M_{\phi}(R)} & =\sup _{t \rightarrow 0}\left\{\frac{1}{\phi(t)} \int_{0}^{t} P^{*}(u) d u\right\}=\sup _{t \rightarrow 0}\left\{\frac{1}{\phi(t)} \int_{0}^{t} h(u) d u\right\}=\sup _{t>0}\left\{\frac{f(t)}{\phi(t)}\right\} \\
& =\operatorname{Max}\left[\sup \left\{\frac{(1-t) \phi(t)}{\phi(t)}\right\}, \sup _{a<t}\left\{\frac{(1-a) \phi(a)}{\phi(t)}\right\}\right]=\sup _{0<t \leqslant a}\{1-t\}=1 .
\end{aligned}
$$


Thus $P \in M_{\phi}(R)$ and $\|P\|_{M_{\phi}(R)}=1$. By Theorem 4.3 [24], $M_{\phi}(R)$ is isometrically isomorphic to $N_{\phi}(R)^{*}$ and $P$ may be identified with $l \in N_{\phi}(R)^{*}$ by

$$
l(j)=\int_{X} P(x) j(x) d \mu(x) \text { for all } j \in N_{\phi}(R) .
$$

Assume now that $N_{\phi}(R)$ is reflexive. Then $U\left(N_{\phi}(R)\right)$ is weakly compact, and $l$ must attain its supremum at some $f_{0}$ in $U\left(N_{\phi}(R)\right)$. It might be assumed that $f_{0} \in \operatorname{Ext} U\left(N_{\phi}(R)\right)$. From the Proposition 3 in the next section the proof of which is self-contained, it follows that there exist $A, B \in \Sigma, f_{0}=c \chi_{A}-c \chi_{B}$ and $c \phi(\mu(A \cup B))=1$.

$$
l\left(f_{0}\right)=\int_{X} P(x) f_{0}(x) d \mu(x)=c \int_{A \cup B}|P(x)| d \mu(x) \leqslant c \int_{0}^{\mu(A \cup B)} P^{*}(t) d t,
$$

the last inequality following from Lemma 3.17 in [25]. Thus

$$
\begin{aligned}
1=l\left(f_{0}\right) & \leqslant c \int_{0}^{\mu(A \cup B)} P^{*}(t) d t=\frac{1}{\phi(\mu(A \cup B))} \int_{0}^{\mu(A \cup B)} h(y) d y=\frac{f(\mu(A \cup B))}{\phi(\mu(A \cup B))} \\
& = \begin{cases}1-\mu(A \cup B) & \text { if } \mu(A \cup B) \leqslant a, \\
\frac{(1-a) \phi(a)}{\phi(\mu(A \cup B))} & \text { if } \mu(A \cup B)>a, \\
& <1,\end{cases}
\end{aligned}
$$

and this is a contradiction. Thus $N_{\phi}(R)$ is not reflexive and therefore $N_{\phi}(E)$ is not reflexive.

In concluding this section we wish to thank the referee for bringing to our notice a paper by DeJonge $\left[9_{2}\right]$ which appeared subsequent to our communication. The Theorem 3.4 in $\left[9_{2}\right]$ is essentially the same as Theorems $2 \mathrm{~A}$ and 3 of this section applied to the special case when $E$ is the real line. The technique adopted by DeJonge is different from the more geometric approach provided here.

6. Extreme points of the unit cell of $N_{\phi}(R)$. In this section the extreme points of $U\left(N_{\phi}(R)\right)$ are completely characterized. For these results it is assumed that either $\mu(X)<\infty$ or $(X, \Sigma, \mu)$ is $\sigma$-finite and $\phi$ is unbounded. Proposition 3 provides a necessary condition for a function $f \in N_{\phi}(R)$ to be an extreme point.

Proposition 3. Let $f \in \operatorname{Ext} U\left(N_{\phi}(R)\right)$, then $|f|$ assumes exactly one value different from 0 .

Proof. Define $g(\xi)=\int_{0}^{\xi} \phi\left(\lambda_{2 f}(y)\right) d y$. Then $g(\infty)=\|2 f\|=2$ and $g(0)=$ 0 . Since $g$ is continuous on $[0, \infty)$, there exists $c, 0<c<\infty$, such that $g(c)=1$, i.e.

$$
\int_{0}^{c} \phi\left(\lambda_{2 f}(y)\right) d y=1 \text { and } \int_{c}^{\infty} \phi\left(\lambda_{2 f}(y)\right) d y=1
$$


Let

and

$$
f_{1}(x)= \begin{cases}2 f(x) & \text { if } 0 \leqslant|2 f(x)|<c, \\ c \operatorname{sign} f(x) & \text { if }|2 f(x)| \geqslant c,\end{cases}
$$

$$
f_{2}(x)= \begin{cases}0 & \text { if } 0 \leqslant|2 f(x)|<c, \\ 2 f(x)-c \operatorname{sign} f(x) & \text { if }|2 f(x)| \geqslant c .\end{cases}
$$

Then $f_{1}, f_{2} \in M_{R}$ and $f=\left(f_{1}+f_{2}\right) / 2$. Let $A=\{x \in X:|2 f(x)| \geqslant c\}$ and $B=$ $\{x \in X:|2 f(x)|<c\}$.

$$
\begin{aligned}
\left\|f_{1}\right\|_{N_{\phi}(R)} & =\int_{0}^{c} \phi^{\circ} \mu[\{x \in B:|2 f(x)|>y\} \cup\{x \in X:|2 f(x)| \geqslant c\}] d y \\
& =\int_{0}^{c} \phi\left(\lambda_{2 f}(y)\right) d y=1 . \\
\left\|f_{2}\right\|_{N_{\phi}(R)} & =\int_{0}^{\infty} \phi^{\circ} \mu[\{x \in A, f(x)>0: 2 f(x)-c>y\} \\
& =\int_{0}^{\infty} \phi^{\circ} \mu[\{x \in A, f(x)>0: 2 f(x)>y+c\} \\
& =\int_{0}^{\infty} \phi^{\circ} \mu\{x \in A:|2 f(x)|>y+c\} d y \\
& =\int_{c}^{\infty} \phi^{\circ} \mu\{x \in A:|2 f(x)|>y\} d y \\
& \left.=\int_{c}^{\infty} \phi(\lambda, f(x)<y,-2 f(x)-c>y\}\right] d y
\end{aligned}
$$

Therefore $f_{1}, f_{2} \in S\left(N_{\phi}(R)\right)$. Since $f$ is an extreme point of $U\left(N_{\phi}(R)\right), f_{1}=$ $f_{2}=f$ : Thus $2 f(x)=0$ for $x \in B$, so that $f=0$ on $B=X \sim A$. Since $c$ sign $f(x)=2 f(x)-c \operatorname{sign} f(x)$ for $x \in A,|f|=c$ on $A$. Thus $|f|$ assumes exactly one value different from 0 .

Considering the positive and negative parts of $f$, and using the preceding proposition, the following corollary obtains:

Corollary. Let $f \in N_{\phi}(R)$. Then $f \in \operatorname{Ext} U\left(N_{\phi}(R)\right)$ if and only if $|f| \in$ Ext $U\left(N_{\phi}(R)\right)$.

Because of this corollary, it is only necessary, in determining $\operatorname{Ext} U\left(N_{\phi}(R)\right)$, 
to consider functions of the form $f=c \chi_{A}$ with $0<c$.

The simplest case to consider is that in which $\mu(A)=\infty$. For $f=c \chi_{A}$ to be an element of $N_{\phi}(R)$, it is necessary that $\phi$ be a bounded function. The next proposition determines when such a function $f$ is an extreme point.

PRoposition 4. Let $f \in S\left(N_{\phi}(R)\right)$ be of the form $f=c \chi_{A}$ with $c>0$ and $\mu(A)=\infty$. Then $f \in \operatorname{Ext} U\left(N_{\phi}(R)\right)$ if and only if $\mu(X \sim A)=0$.

Proof. Suppose first that $f \in \operatorname{Ext} U\left(N_{\phi}(R)\right)$ but $\mu(X \sim A)>0$. Let $f_{1}=$ $c \chi_{A}+c \chi_{X-A}$ and $f_{2}=c \chi_{A}-c \chi_{X-A}$. Then $f=\left(f_{1}+f_{2}\right) / 2$ and $f_{1} \neq f_{2}$ since $\mu(X \sim A)>0$. By direct computation it is verified that $\left\|f_{2}\right\|=\left\|\left|f_{2}\right|\right\|=\left\|f_{1}\right\|=$ 1. Thus $f_{1}, f_{2} \in S\left(N_{\phi}(R)\right)$ and this is a contradiction.

Next suppose that $\mu(X-A)=0$ so that $f=c \chi_{X}$, but $f \notin \operatorname{Ext} U\left(N_{\phi}(R)\right)$. Thus there exist $f_{1}, f_{2} \in S\left(N_{\phi}(R)\right), f_{1} \neq f_{2}$, and $f=\left(f_{1}+f_{2}\right) / 2$.

Assume first that $\mu\left\{x \in X: f_{1}(x) \neq f_{2}(x)\right\}=\infty$ and let

$$
A_{1}=\left\{x \in X:\left|f_{1}(x)\right|<\left|f_{2}(x)\right|\right\} \text { and } A_{2}=\left\{x \in X:\left|f_{1}(x)\right|>\left|f_{2}(x)\right|\right\} \text {. }
$$

Then either $\mu\left(A_{1}\right)=\infty$ or $\mu\left(A_{2}\right)=\infty$. It may be assumed that $\mu\left(A_{1}\right)=\infty$ so that $\left|f_{2}(x)\right|>c$ for $x \in A_{1}$. Thus there exists $n \geqslant 1$ and $B \subset A_{1}, 0<\mu(B)$, such that $\left|f_{2}(x)\right|>c+1 / n$ for $x \in B$. Then

$$
\begin{aligned}
\left\|f_{2}\right\| & =\int_{0}^{\infty} \phi\left(\lambda_{f_{2}}(y)\right) d y \geqslant \int_{0}^{c} \phi\left(\mu\left(A_{1}\right)\right) d y+\int_{c}^{c+1 / n} \phi(\mu(B)) d y \\
& =c \phi\left(\mu\left(A_{1}\right)\right)+\phi(\mu(B)) / n=1+\phi(\mu(B)) / n>1,
\end{aligned}
$$

thus contradicting $f_{2} \in S\left(N_{\phi}(R)\right)$.

Next assume that $0<\mu\left\{x \in X: f_{1}(x) \neq f_{2}(x)\right\}<\infty$. Then, if $D=\{x \in X$ : $f_{1}(x)=f_{2}(x)=c$, it follows that $\mu(D)=\infty$. If $A_{1}$ and $A_{2}$ are defined as above, then either $0<\mu\left(A_{1}\right)$ or $0<\mu\left(A_{2}\right)$. Assuming that $\mu\left(A_{1}\right)>0$, as before there exists $B^{\prime} \subset A_{1}, 0<\mu\left(B^{\prime}\right)$, such that $\left|f_{2}(x)\right|>c+1 / n^{\prime}$ for $x \in B^{\prime}$. Then

$$
\begin{aligned}
\left\|f_{2}\right\| & =\int_{0}^{\infty} \phi\left(\lambda_{f_{2}}(y)\right) d y>\int_{0}^{c} \phi(\mu(D)) d y+\int_{c}^{c+1 / n^{\prime}} \phi\left(\mu\left(B^{\prime}\right)\right) d y \\
& =c \phi(\mu(D))+\phi\left(\mu\left(B^{\prime}\right)\right) / n^{\prime}=1+\phi\left(\mu\left(B^{\prime}\right)\right) / n^{\prime}>1,
\end{aligned}
$$

again contradicting $f_{2} \in S\left(N_{\phi}(R)\right)$. Thus $\mu(X \sim A)=0$ implies that $f \in$ Ext $U\left(N_{\phi}(R)\right)$.

The remaining propositions in this section are concerned with functions of the form $f=c \chi_{A}$ with $0<c$ and $\mu(A)<\infty$.

If $A \in \Sigma$, then $\mu(A)$ satisfies condition (*) if there exists an $\alpha>\mu(A)$ such that $\phi$ is not constant in any subinterval of the interval $[0, \alpha]$.

Proposition 5. If $f \in \operatorname{Ext} U\left(N_{\phi}(R)\right)$ is of the form $f=c \chi_{A}$ with $0<c$ and $\mu(A)<\infty$, then either $\mu(X-A)=0$ or $\mu(A)$ satisfies condition (*). 
The proof of this proposition makes use of the property in Proposition 1, and arguments similar to the preceding proposition. Hence the proof is omitted.

The rest of this section is concerned with the converse of Propositions 3 and 5. These results are heavily dependent on the nature of the graph of $\phi$. In particular, it is possible that any or all of the following conditions may hold: (i) $\lim _{x \rightarrow \infty} \phi(x)<\infty$, (ii) there exist $x_{0}$ and $\alpha$ with $0<x_{0}, 0<\alpha$ such that $\phi(x)=\alpha$ for $x \geqslant x_{0}$, (iii) there exist $k$ and $x_{0}$ with $0<k, 0<x_{0}$ such that $\phi(x)=k x$ for $x \in\left[0, x_{0}\right]$. For convenience, the converse is stated in two propositions, 6 and 7, and the extreme point characterization is summarized in Theorem 4.

PROPOSITION 6. Let $f \in S\left(N_{\phi}(R)\right)$ be of the form $f=c \chi_{A}$ with $c>0$ and $\mu(A)<\infty$. If there does not exist any $k>0$ such that $\phi(x)=k x$ for $x \in[0$, $\mu(A)]$, then the requirement that either $\mu(X-A)=0$ or $\mu(A)$ satisfies (*) is sufficient as well as necessary for $f$ to be an element of $\operatorname{Ext} U\left(N_{\phi}(R)\right)$.

Proposition 7. Let $f \in S\left(N_{\phi}(R)\right)$ be of the form $f=c \chi_{A}$ with $c>0$ and assume that $\phi(x)=k x$ for $x \in[0, \mu(A)]$. Then $f \in \operatorname{Ext} U\left(N_{\phi}(R)\right)$ if and only if $A$ is an atom of $(X, \Sigma, \mu)$ which meets the requirement that either $\mu(X \sim A)=0$ or $\mu(A)$ satisfies (*).

The following lemmas are required in the proofs of the above propositions. The proofs of the lemmas are very similar, and use the same techniques. As a typical case, proof of Lemma 6 is provided. The proofs of others are omitted.

LEMMA 6. If $f \in N_{\phi}(R)$ and there exists $k>0$ such that, for all $y \geqslant 0$, $\lambda_{f}(y)=0$ or $\lambda_{f}(y)=k$, then $f=\alpha \chi_{A_{1}}-\alpha \chi_{A_{2}}$ for some $\alpha>0$. If $A_{1} \cap A_{2}=$ $\varnothing$, then $\mu\left(A_{1} \cup A_{2}\right)=k$.

Proof. Let $\alpha=\sup \left\{y \geqslant 0: \lambda_{f}(y)=k\right\}$. Then, since $f \in N_{\phi}(R)$, it follows that $0<\alpha<\infty$. Let $B=\{x \in X:|f(x)|>\alpha\}$ and suppose $\mu(B)>0$. Since $B=$ $\bigcup_{n \geqslant 1}\{x \in X:|f(x)|>\alpha+1 / n\}$, there exists $n_{0}$ such that $\mu\{x \in X:|f(x)|>\alpha+$ $\left.1 / n_{0}\right\}=\lambda_{f}\left(\alpha+1 / n_{0}\right)>0$. This implies that $\lambda_{f}\left(\alpha+1 / n_{0}\right)=k$, thus contradicting the definition of $\alpha$.

Let $B^{\prime}=\{x \in X: 0<|f(x)|<\alpha\}$ and suppose $\mu\left(B^{\prime}\right)>0$. Then, arguing as above, there exists $n_{1}$ such that $\mu\left\{x \in X: 0<|f(x)|<\alpha-1 / n_{1}\right\}>0$. Further

$$
\begin{aligned}
\{x \in X:|f(x)|>0\} \supseteq\left\{x \in X: 0<|f(x)|<\alpha-1 / n_{1}\right\} \\
\cup\left\{x \in X:|f(x)|>\alpha-1 / n_{1}\right\} .
\end{aligned}
$$

Therefore

$$
\begin{aligned}
k & =\lambda_{f}(0) \geqslant \mu\left\{x \in X: 0<|f(x)|<\alpha-1 / n_{1}\right\}+\lambda_{f}\left(\alpha-1 / n_{1}\right) \\
& =\mu\left\{x \in X: 0<|f(x)|<\alpha-1 / n_{1}\right\}+k>k,
\end{aligned}
$$


a contradiction. Hence $f=\alpha \chi_{A_{1}}-\alpha \chi_{A_{2}}$ and $\lambda_{f}(0)=\mu\left(A_{1} \cup A_{2}\right)=k$.

LEMMA 7. If $f \in N_{\phi}(R)$ is such that there exist $y_{1}, y_{2}, 0 \leqslant y_{1}<y_{2}$, with $\infty>\lambda_{f}(0) \geqslant \lambda_{f}\left(y_{1}\right)>\lambda_{f}\left(y_{2}\right)>0$ and if $A \subset[0, \infty)$ is a set of Lebesgue measure 0 , then there exist $y_{1}^{\prime}, y_{2}^{\prime}, 0 \leqslant y_{1}^{\prime}<y_{2}^{\prime}$, such that $y_{i}^{\prime} \in[0, \infty)-A$ and $\lambda_{f}\left(y_{1}^{\prime}\right)>$ $\lambda_{f}\left(y_{2}^{\prime}\right)>0$.

LEMMA 8. Let $f \in S\left(N_{\phi}(R)\right)$ be of the form $f=c \chi_{A}$ with $c>0$. If there exist $f_{1}, f_{2} \in S\left(N_{\phi}(R)\right), f_{1} \neq f_{2}$ with support $\left(f_{i}\right) \subset A, f=\left(f_{1}+f_{2}\right) / 2$, and if $\left|f_{i}(x)\right|=k_{i} x_{c_{i}}(x), i=1,2$, then there exists $k>0$ such that $\phi(x)=k x$ for $x \in$ $[0, \mu(A)]$.

Proof of Proposition 6. By Proposition 5, it is enough to show that if either $\mu(X \sim A)=0$ or $\mu(A)$ satisfies (*), then $f \in \operatorname{Ext} U\left(N_{\phi}(R)\right)$. Assume that $f \notin \operatorname{Ext} U\left(N_{\phi}(R)\right)$ so that there exist $f_{1}, f_{2} \in S\left(N_{\phi}(R)\right)$ with $f_{1} \neq f_{2}$ and $f=$ $\left(f_{1}+f_{2}\right) / 2$. For $E \in \Sigma$, define

$$
m(E)=\phi(\mu(A)) \mu(A \cap E) / \mu(A) .
$$

It is verified that $m \in M$ and therefore $m$ represents a continuous linear functional on $N_{\phi}(R)$. Further by direct computation, $\|m\|=1$. Also, since $\int_{X} f d m=1$, $m$ represents a support functional for $f$. Define $l(g)=\int_{X} g d m$ for $g \in N_{\phi}(R)$. Then $l \in N_{\phi}(R)^{*}$ and $\|l\|=1$.

$$
1=\int_{X} f d m=\frac{1}{2}\left(\int_{X} f_{1} d m+\int_{X} f_{2} d m\right),
$$

and since $\left|l\left(f_{i}\right)\right| \leqslant\|l\|\left\|f_{i}\right\|=1$, i.e., $\left|\int_{X} f_{i} d m\right| \leqslant 1$, it follows that $\int_{X} f_{i} d m=1$. Further, $\left|l\left(\left|f_{i}\right|\right)\right| \leqslant\|l\|\left\|f_{i}\right\|=1$, which combined with $l\left(\left|f_{i}\right|\right)=\int_{X}\left|f_{i}\right| d m \geqslant$ $\int_{X} f_{i} d m=1$, yields that $\int_{X}\left|f_{i}\right| d m=1$.

It is now claimed that it might be assumed that the set $\left\{x \in X:\left|f_{i}(x)\right|>\right.$ $0\} \subset A$. If $\mu(X \sim A)=0$, then this is clear. Otherwise, $\left\|f_{i} \chi_{A}\right\| \leqslant\left\|f_{i}\right\|=1$. But $l\left(f_{i} \chi_{A}\right)=\int_{A} f_{i} d m=\int_{X} f_{i} d m=1$, so that $1=\left|l\left(f_{i} \chi_{A}\right)\right| \leqslant\left\|f_{i} \chi_{A}\right\| \leqslant 1$ which im. plies that $\left\|f_{i} \chi_{A}\right\|=1$. Since $f_{1} \chi_{A}+f_{2} \chi_{A}=2 f$, it need only be shown that $f_{1} \chi_{A} \neq f_{2} \chi_{A}$.

Assume that $f_{1} \chi_{A}=f_{2} \chi_{A}$. Then $f_{1}=f_{2}=c$ on $A$, and there exists $B \in$ $\Sigma, A \cap B=\varnothing, \mu(B)>0$, such that $f_{1} \neq f_{2}$ on $B$. Thus $f_{1}=-f_{2}$ on $B$. Choose $B^{\prime} \subset B, \mu\left(B^{\prime}\right)>0$, such that $\left|f_{i}(x)\right|>\epsilon>0$ for $x \in B^{\prime}$, and for convenience assume that $\epsilon<c$. Then

$$
\begin{aligned}
\left\|f_{i}\right\|=\int_{0}^{\infty} \phi\left(\lambda_{f_{i}}(y)\right) d y & \geqslant \int_{0}^{\epsilon} \phi\left(\mu(A)+\mu\left(B^{\prime}\right)\right) d y+\int_{\epsilon}^{c} \phi(\mu(A)) d y \\
& =\epsilon \phi\left(\mu(A)+\mu\left(B^{\prime}\right)\right)+(c-\epsilon) \phi(\mu(A)) .
\end{aligned}
$$

But since there exists $\alpha>\mu(A)$ such that $\phi$ is not constant in any subinterval of $[0, \alpha]$, it follows that $\phi\left(\mu(A)+\mu\left(B^{\prime}\right)\right)>\phi(\mu(A))$. Therefore 


$$
\left\|f_{i}\right\|>\epsilon \phi(\mu(A))+(c-\epsilon) \phi(\mu(A))=1,
$$

contradicting $f_{i} \in S\left(N_{\phi}(R)\right)$. Thus $f_{1} \chi_{A} \neq f_{2} \chi_{A}$, and it may thus be assumed that $\left\{x \in X:\left|f_{i}(x)\right|>0\right\} \subset A$.

Now

$$
1=\left\|f_{i}\right\|=\int_{0}^{\infty} \phi\left(\lambda_{f_{i}}(y)\right) d y=\int_{0}^{\infty} \phi\left(\frac{\lambda_{f_{i}}(y)}{\mu(A)} \mu(A)\right) d y .
$$

But $\left\{x \in X:\left|f_{i}(x)\right|>0\right\} \subset A$ implies that $\lambda_{f_{i}}(0) \leqslant \mu(A)$ so $\lambda_{f_{i}}(y) \leqslant \mu(A)$ for all $y \geqslant 0$. Thus $\lambda_{f_{i}}(y) / \mu(A) \leqslant 1$ and therefore

$$
\begin{aligned}
\int_{0}^{\infty} \phi\left(\frac{\lambda_{f_{i}}(y)}{\mu(A)} \mu(A)\right) d y & \geqslant \int_{0}^{\infty} \frac{\lambda_{f_{i}}(y)}{\mu(A)} \phi(\mu(A)) d y=\frac{\phi(\mu(A))}{\mu(A)} \int_{0}^{\infty} \lambda_{f_{i}}(y) d y \\
& =\int_{0}^{\infty} m\left\{x \in X:\left|f_{i}(x)\right|>y\right\} d y=\int_{X}\left|f_{i}\right| d m=1 .
\end{aligned}
$$

Therefore

$$
\int_{0}^{\infty}\left[\phi\left(\lambda_{f_{i}}(y)\right)-\frac{\phi(\mu(A))}{\mu(A)} \lambda_{f_{i}}(y)\right] d y=0,
$$

and since the integrand is $\geqslant 0$, this implies that

$$
\phi\left(\lambda_{f_{i}}(y)\right)=\frac{\phi(\mu(A))}{\mu(A)} \lambda_{f_{i}}(y) \text { for } y \text {-a.e. }
$$

Next it is shown that at least one of the functions $\lambda_{f_{1}}, \lambda_{f_{2}}$ has a range consisting of more than one positive value. That is, there exist $y_{1}, y_{2}$ with $y_{1}<y_{2}$ such that either $\lambda_{f_{1}}\left(y_{1}\right)>\lambda_{f_{1}}\left(y_{2}\right)>0$ or $\lambda_{f_{2}}\left(y_{1}\right)>\lambda_{f_{2}}\left(y_{2}\right)>0$. If this is not true, then, for some $\alpha_{1}, \alpha_{2}>0, \lambda_{f_{i}}(y)=0$ or $\lambda_{f_{i}}(y)=\alpha_{i}$ for all $y \geqslant 0$. Thus, by Lemma $6, f_{1}=k_{1} \chi_{A_{1}}-k_{1} \chi_{A_{2}}$ and $f_{2}=k_{2} \chi_{B_{1}}-k_{2} \chi_{B_{2}}$. But Lemma 8 then implies that $\phi(x)=k x$ for $x \in[0, \mu(A)]$, and this is a contradiction. Therefore there exists such a pair $y_{1}, y_{2}$ for $f_{1}$ or $f_{2}$, and it may be assumed that $\lambda_{f_{1}}\left(y_{1}\right)>\lambda_{f_{1}}\left(y_{2}\right)>0$.

Now let $B=\left\{y \geqslant 0: \phi\left(\lambda_{f_{1}}(y)\right) \neq \phi(\mu(A)) \lambda_{f_{1}}(y) / \mu(A)\right\}$ so that $B$ has Lebesgue measure 0 . Since $\lambda_{f_{1}}(0) \leqslant \mu(A)<\infty$, Lemma 7 may be applied to conclude that there exist $y_{1}^{\prime}, y_{2}^{\prime} \in[0, \infty) \sim B$ such that $\lambda_{f_{1}}\left(y_{1}^{\prime}\right)>\lambda_{f_{1}}\left(y_{2}^{\prime}\right)>0$. Thus

$$
\phi\left(\lambda_{f_{1}}\left(y_{i}^{\prime}\right)\right)=\phi(\mu(A)) \lambda_{f_{1}}\left(y_{i}^{\prime}\right) / \mu(A) .
$$

Now by Proposition 1, $\phi(x)=\phi(\mu(A)) x / \mu(A)$ for $x \in[0, \mu(A)]$ and this is a contradiction. Therefore $f \in \operatorname{Ext} U\left(N_{\phi}(R)\right)$.

The proof of the Proposition 7 is similar in content to the preceding proof, and details are omitted.

The next theorem summarizes the results characterizing $\operatorname{Ext} U\left(N_{\phi}(R)\right)$.

THEOREM 4. Let $f \in S\left(N_{\phi}(R)\right)$. For $f$ to be an element of $\operatorname{Ext} U\left(N_{\phi}(R)\right)$ 
it is necessary that $f=c \chi_{A}-c \chi_{B}$ with $0<c$ and $0<\mu(A \cup B)$. Let $f$ be of this form and assume that $A \cap B=\varnothing$. $=0$.

(i) If $\mu(A \cup B)=\infty$, then $f \in \operatorname{Ext} U\left(N_{\phi}(R)\right)$ if and only if $\mu(X-(A \cup B))$

(ii) If $\mu(A \cup B)<\infty$, then

(a) If there exists $k>0$ such that $\phi(x)=k x$ for $x \in[0, \mu(A \cup B)]$, $f \in \operatorname{Ext} U\left(N_{\phi}(R)\right)$ if and only if $A \cup B$ is an atom of $(X, \Sigma, \mu)$ and either $\mu(X-(A \cup B))=0$ or $\mu(A \cup B)$ satisfies (*).

(b) If there does not exist such a $k$, then $f \in \operatorname{Ext} U\left(N_{\phi}(R)\right)$ if and only if either $\mu(X-(A \cup B))=0$ or $\mu(A \cup B)$ satisfies (*).

Corollary. Let $\phi(x)=x^{s}$ for some $s \in(0,1)$. Then $f \in S\left(N_{\phi}(R)\right)$ is an extreme point of $U\left(N_{\phi}(R)\right)$ if and only if $f=c \chi_{A}-c \chi_{B}$.

7. Extreme points of the unit cell of $N_{\phi}(E)$. In this section the set of extreme points of the unit cell of $N_{\phi}(E)$ are discussed. When $E$ is a separable Banach space a complete characterization is obtained. The techniques are similar to Sundaresan [28], and Johnson [ $\left.9_{1}\right]$. Throughout this section $E$ is a Banach space unless otherwise specified, and $\mu(X)<\infty$ or $\mu$ is $\sigma$-finite, and $\phi$ is unbounded. The first proposition is a direct generalization of Proposition 3.

Proposition 8. If $f \in \operatorname{Ext} U\left(N_{\phi}(E)\right)$, then $\|f(x)\|=c \chi_{A}(x)$ for some $A \in$ $\Sigma$, and $c>0$.

The proof is the same as in the case of Proposition 3 except $|f(x)|$ is replaced by $\|f(x)\|$, and sign $f(x)$ is replaced by $f(x) /\|f(x)\|$.

The next proposition determines the functions $f \in S\left(N_{\phi}(E)\right),\|f(x)\|=$ $c \chi_{A}(x)$ with $\mu(A)=\infty$, which are in $\operatorname{Ext} U\left(N_{\phi}(E)\right)$.

In this section the following notation is adopted. $U(E)$ is the unit cell of $E$ and $C=\left\{f \mid f \in N_{\phi}(E), f(x) \in U(E)\right.$, for all $\left.x \in X\right\}$. $C$ is a convex subset of $N_{\phi}(E)$. If $f \in N_{\phi}(E)$, let $P f$ be the function in $N_{\phi}(E)$ such that $P f(x)=$ $f(x) /\|f(x)\|$, if $f(x) \neq 0$, and $P f(x)=0$ if $f(x)=0$. Note that $P f \in C$. The same symbol \|\| is used for various norms entering the discussion as there is no possibility of confusion.

Proposition 9. Let $f \in S\left(N_{\phi}(E)\right)$ be such that $\|f(x)\|=c \chi_{A}(x)$ with $c>$ 0 and $\mu(A)=\infty$. Then $f \in \operatorname{Ext} U\left(N_{\phi}(E)\right)$ if and only if $\mu(X-A)=0$ and, Pf is an extreme point of $C$.

Proof. Suppose $f \in$ Ext $U\left(N_{\phi}(E)\right)$. Replacing in the proof of Proposition $4, c \chi_{A}(x), c \chi_{X \sim A}(x)$ respectively by $f(x) \chi_{A}(x)$ and $x_{0} \chi_{X \sim A}(x)$ where $x_{0} \in E$ such that $\left\|x_{0}\right\|=c$, it is verified that $\mu(X \sim A)=0$. 
Next it must be shown that if $\mu(X \sim A)=0$ and $P f \in \operatorname{Ext} C$, then $f \in$ $\operatorname{Ext} U\left(N_{\phi}(E)\right)$.

Suppose that the above property holds so that $\|f(x)\|=c$ for $x \in X$, but $f \notin \operatorname{Ext} U\left(N_{\phi}(E)\right)$. Thus there exist $f_{1}, f_{2} \in S\left(N_{\phi}(E)\right), f_{1} \neq f_{2}$, and $f=$ $\left(f_{1}+f_{2}\right) / 2$. Define

$$
\begin{aligned}
& A_{1}=\left\{x \in X:\left\|f_{1}(x)\right\|=\left\|f_{2}(x)\right\|, f_{1}(x) \neq f_{2}(x)\right\}, \\
& A_{2}=\left\{x \in X:\left\|f_{1}(x)\right\|<\left\|f_{2}(x)\right\|\right\}
\end{aligned}
$$

and

$$
A_{3}=\left\{x \in X:\left\|f_{2}(x)\right\|<\left\|f_{1}(x)\right\|\right\} .
$$

Then $\left\|f_{1}(x)\right\| \geqslant c$ for $x \in A_{1},\left\|f_{2}(x)\right\|>c$ for $x \in A_{2}$, and $\left\|f_{1}(x)\right\|>c$ for $x \in A_{3}$. Also, $\mu\left(A_{1} \cup A_{2} \cup A_{3}\right)>0$ since $f_{1} \neq f_{2}$.

If $\mu\left(A_{1} \cup A_{2} \cup A_{3}\right)=\infty$, then $\mu\left(A_{2}\right)<\infty$ and $\mu\left(A_{3}\right)<\infty$. For suppose $\mu\left(A_{2}\right)=\infty$. Choose $A_{2}^{\prime} \subset A_{2}, \mu\left(A_{2}^{\prime}\right)>0$ such that $\left\|f_{2}(x)\right\|>c+1 / n$ for $x \in$ $A_{2}^{\prime}$. Then

$$
\begin{aligned}
\left\|f_{2}\right\| & =\int_{0}^{\infty} \phi\left(\lambda_{f_{2}}(y)\right) d y \geqslant \int_{0}^{c} \phi\left(\mu\left(A_{2}\right)\right) d y+\int_{c}^{c+1 / n} \phi\left(\mu\left(A_{2}^{\prime}\right)\right) d y \\
& =c \phi(\mu(X))+\phi\left(\mu\left(A_{2}^{\prime}\right)\right) / n>1,
\end{aligned}
$$

contradicting $f_{2} \in S\left(N_{\phi}(E)\right)$. A similar argument shows that $\mu\left(A_{3}\right)<\infty$. Thus $\mu\left(A_{1}\right)=\infty$, and, arguing as above, it then follows that $\left\|f_{i}(x)\right\|=c$ for $x \in A_{1}$. Define

$$
f_{i}^{\prime}(x)= \begin{cases}f(x) & \text { for } x \in X-A_{1}, \\ f_{i}(x) & \text { for } x \in A_{1} .\end{cases}
$$

Then $f=\left(f_{1}^{\prime}+f_{2}^{\prime}\right) / 2, f_{1}^{\prime} \neq f_{2}^{\prime}$, and $\left\|f_{i}^{\prime}(x)\right\|=c \chi_{X}(x)$, and this is a contradiction.

Next, if $\mu\left(A_{1} \cup A_{2} \cup A_{3}\right)<\infty$ so that $\mu\left(X-\left(A_{1} \cup A_{2} \cup A_{3}\right)\right)=\infty$, then $\mu\left(A_{2}\right)=\mu\left(A_{3}\right)=0$. For suppose $\mu\left(A_{2}\right)>0$. Choose $A_{2}^{\prime \prime} \subset A_{2}, \mu\left(A_{2}^{\prime \prime}\right)>0$ such that $\left\|f_{2}(x)\right\|>c+1 / n$ for $x \in A_{2}^{\prime \prime}$. Then

$$
\begin{aligned}
\left\|f_{2}\right\| & =\int_{0}^{\infty} \phi\left(\lambda_{f_{2}}(y)\right) d y \\
& \geqslant \int_{0}^{c} \phi\left(\mu\left(X-\left(A_{1} \cup A_{2} \cup A_{3}\right)\right)\right) d y+\int_{c}^{c+1 / n} \phi\left(\mu\left(A_{2}^{\prime \prime}\right)\right) d y \\
& =\int_{0}^{c} \phi(\mu(X)) d y+\phi\left(\mu\left(A_{2}^{\prime \prime}\right)\right) / n=c \phi(\mu(X))+\phi\left(\mu\left(A_{2}^{\prime \prime}\right)\right) / n>1,
\end{aligned}
$$

contradicting $f_{2} \in S\left(N_{\phi}(E)\right)$. The same argument applied to $A_{3}$ shows that $\mu\left(A_{3}\right)=0$ as well. A similar argument shows that $\left\|f_{i}(x)\right\|=c$ for $x \in A_{1}$, and it therefore follows that $\left\|f_{i}(x)\right\|=c$ for $x \in X$, and $P f \in$ Ext $C$, a contradiction.

The remaining propositions of this part of the section are concerned with 
functions $f \in S\left(N_{\phi}(E)\right)$ such that $\|f(x)\|=c \chi_{A}(x)$ with $\mu(A)<\infty$.

It is recalled that if $A \in \Sigma$, then $\mu(A)$ satisfies condition (*) if there exists an $\alpha>\mu(A)$ such that $\phi$ is not constant in any subinterval of the interval $[0, \alpha]$.

Proposition 10. If $f \in \operatorname{Ext} U\left(N_{\phi}(E)\right)$ and $\|f(x)\|=c \chi_{A}(x)$ with $\mu(A)<$ $\infty$, then either $\mu(X \sim A)=0$ or $\mu(A)$ satisfies (*).

Proof. Assume that $\mu(X \sim A)>0$ and, for all $\alpha>\mu(A), \phi$ is constant in some subinterval of the interval $[0, \alpha]$. Therefore, by Proposition $1, \phi(x)=$ $\phi(\mu(A))$ for all $x \geqslant \mu(A)$. Let

$$
f_{1}(x)=f(x) \chi_{A}(x)+x_{0} \chi_{X \sim A}(x) \text { and } f_{2}(x)=f(x) \chi_{A}(x)-x_{0} \chi_{X \sim A}(x)
$$

where $\left\|x_{0}\right\|=c$. Then $f=\left(f_{1}+f_{2}\right) / 2$ and $f_{1} \neq f_{2}$.

$$
\begin{aligned}
\left\|f_{1}\right\| & =\left\|f_{2}\right\|=\int_{0}^{\infty} \phi\left(\lambda_{f_{2}}(y)\right) d y=\int_{0}^{c} \phi(\mu(X)) d y \\
& =c \phi(\mu(A)+\mu(X \sim A))=c \phi(\mu(A))=1 .
\end{aligned}
$$

Thus $f_{1}, f_{2} \in S\left(N_{\phi}(E)\right)$, contradicting $f \in \operatorname{Ext} U\left(N_{\phi}(E)\right)$.

The following two propositions complete the chracterization of Ext $U\left(N_{\phi}(E)\right)$. This characterization is summarized in Theorem 5 .

Proposition 11. Let $f \in S\left(N_{\phi}(E)\right)$ be such that $\|f(x)\|=c \chi_{A}(x)$ with $\mu(A)<\infty$ and assume that there does not exist any $k>0$ such that $\phi(x)=k x$ for $x \in[0, \mu(A)]$. Then $f \in \operatorname{Ext} U\left(N_{\phi}(E)\right)$ if and only if

(i) $P f \in \operatorname{Ext} C$,

(ii) either $\mu(X \sim A)=0$ or $\mu(A)$ satisfies (*).

PROPOSITION 12. Let $f \in S\left(N_{\phi}(E)\right)$ be such that $\|f(x)\|=c \chi_{A}(x)$ and assume that $\phi(x)=k x$ for $x \in[0, \mu(A)]$. Then $f \in \operatorname{Ext} U\left(N_{\phi}(E)\right)$ if and only if

(i) $f(x)=x_{0} \chi_{A}(x)$ with $x_{0} /\left\|x_{0}\right\| \in \operatorname{Ext} U(E)$,

(ii) $A$ is an atom of $(X, \Sigma, \mu)$, and

(iii) either $\mu(X \sim A)=0$ or $\mu(A)$ satisfies (*).

The next three lemmas are required in the proofs of these two propositions.

Lemma 9. If $f \in N_{\phi}(E), f \neq 0$, and there exists $k>0$ such that for all $y>$ $0, \lambda_{f}(y)=0$ or $\lambda_{f}(y)=k$, then $\|f(x)\|=c \chi_{A}(x)$ for some $A \in \Sigma$.

Proof. The proof of this lemma is the same as the proof of Lemma 6.

LEMmA 10. If $f \in N_{\phi}(E)$ is such that there exist $y_{1}, y_{2}, 0 \leqslant y_{1}<y_{2}$, with $0<\lambda_{f}\left(y_{2}\right)<\lambda_{f}\left(y_{1}\right) \leqslant \lambda_{f}(0)<\infty$ and if $A \subset[0, \infty)$ is a set of Lebesgue measure zero, then there exist $y_{1}^{\prime}, y_{2}^{\prime}, 0 \leqslant y_{1}^{\prime}<y_{2}^{\prime}$, such that $y_{1}^{\prime} \in[0, \infty) \sim A$ and $0<$ $\lambda_{f}\left(y_{2}^{\prime}\right)<\lambda_{f}\left(y_{1}^{\prime}\right)$. 
Proof. The proof of this lemma is the same as the proof of Lemma 8 .

LEMMA 11. Let $f \in S\left(N_{\phi}(E)\right)$ be such that $\|f(x)\|=c \chi_{A}(x)$. If there exist $f_{1}, f_{2} \in S\left(N_{\phi}(E)\right), f_{1} \neq f_{2}, f=\left(f_{1}+f_{2}\right) / 2$, and $\left\|f_{i}(x)\right\|=k_{i} \chi_{A_{i}}(x)$ with $A_{i} \subset A$, then either there exists $k>0$ such that $\phi(x)=k x$ for $x \in[0, \mu(A)]$ or $k_{1}=$ $k_{2}=c$ and $A_{1}=A_{2}=A$.

Proof. If $\mu\left(A_{1} \sim A_{2}\right)>0$, then $k_{1}=2 c$. If also $\mu\left(A_{2} \sim A_{1}\right)=0$, then $A_{1}=A$ so that $\left\|f_{1}\right\|=2$, contradicting $f_{1} \in S\left(N_{\phi}(E)\right)$. Therefore $\mu\left(A_{2} \sim A_{1}\right)>$ 0 , so that also $k_{2}=2 c$. Thus

$$
\left\|f_{i}\right\|=k_{i} \phi\left(\mu\left(A_{i}\right)\right)=c \phi(\mu(A)) \text {, }
$$

and it follows that $\phi\left(\mu\left(A_{i}\right)\right)=\phi(\mu(A)) / 2$ for $i=1,2$.

$$
\phi(\mu(A))=\phi\left(\mu\left(A_{1} \cup A_{2}\right)\right) \leqslant \phi\left(\mu\left(A_{1}\right)+\mu\left(A_{2}\right)\right) \leqslant \phi(\mu(A)),
$$

the last inequality following from Proposition 1. Therefore $\phi\left(\mu\left(A_{1}\right)+\mu\left(A_{2}\right)\right)=$ $\phi\left(\mu\left(A_{1}\right)\right)+\phi\left(\mu\left(A_{2}\right)\right)$ so that by Proposition 1 , there exists $k>0$ such that $\phi(x)=k x$ for $x \in[0, \mu(A)]$.

If $\mu\left(A_{1} \sim A_{2}\right)=0$, then, by the above argument, $\mu\left(A_{2} \sim A_{1}\right)=0$, so that $A_{1}=A_{2}=A$. Therefore $\left\|f_{i}\right\|=k_{i} \phi(\mu(A))=c \phi(\mu(A))$ so that $k_{1}=k_{2}=c$.

Proof of Proposition 11. By Proposition 10 it is enough to show that (i) and (ii) are sufficient to imply that $f \in \operatorname{Ext} U\left(N_{\phi}(E)\right)$. Assume that $f \notin$ Ext $U\left(N_{\phi}(E)\right)$, so that there are $f_{1}, f_{2} \in S\left(N_{\phi}(E)\right)$ with $f_{1} \neq f_{2}$ and $f=$ $\left(f_{1}+f_{2}\right) / 2$. For $B \in \Sigma$, define

$$
m(B)=\frac{\phi(\mu(A))}{\mu(A)} \mu(A \cap B) .
$$

It is verified that $m \in M$, and $\|m\|=1$. Also, since $\int_{X} f d m=1, m$ represents a support functional for $f$. Define

$$
l(g)=\int_{X} g d m \quad \text { for } g \in N_{\phi}(R) .
$$

Then $l \in N_{\phi}(R)^{*}$ and $\|l\|=1$.

$$
\begin{aligned}
2 & =2 \int_{X} \tilde{f} d m=\int_{X}\left\|f_{1}(x)+f_{2}(x)\right\| d m(x) \\
& \leqslant \int_{X}\left(\widetilde{f_{1}+f_{2}}\right) d m \leqslant \int_{X}\left(\tilde{f}_{1}+\widetilde{f}_{2}\right) d m \\
& =l\left(\tilde{f}_{1}\right)+l\left(\tilde{f}_{2}\right) \leqslant\left\|\tilde{f}_{1}\right\|+\left\|\tilde{f}_{2}\right\|=2 .
\end{aligned}
$$

It therefore follows that $\int_{X} \widetilde{f}_{i} d m=1$.

It is now claimed that $\left\{x \in X:\left\|f_{i}(x)\right\|>0\right\} \subset A$ can be assumed. If $\mu(X \sim A)=0$, then this is clear. Otherwise, since $2 f=f_{1} \chi_{A}+f_{2} \chi_{A}$ it is veri- 
fied that $2=2\|f\|=\left\|f_{1} \chi_{A}+f_{2} \chi_{A}\right\| \leqslant\left\|f_{1} \chi_{A}\right\|+\left\|f_{2} \chi_{A}\right\| \leqslant 2$. Thus $\left\|f_{i} \chi_{A}\right\|=1$, and it need only be shown that $f_{1} \chi_{A} \neq f_{2} \chi_{A}$.

Assume that $f_{1} \chi_{A}=f_{2} \chi_{A}$. Then there exists $B \in \Sigma, A \cap B=\varnothing, \mu(B)>0$, such that $f_{1} \neq f_{2}$ on $B$. Thus $f_{1}=-f_{2}$ on $B$. Choose $B^{\prime} \subseteq B, \mu\left(B^{\prime}\right)>0$, such that $\left\|f_{i}(x)\right\|>\epsilon>0$ for $x \in B^{\prime}$, and for convenience assume that $\epsilon<c$. Then

$$
\begin{aligned}
\left\|f_{i}\right\| & =\int_{0}^{\infty} \phi\left(\lambda_{f_{i}}(y)\right) d y \geqslant \int_{0}^{\epsilon} \phi\left(\mu(A)+\mu\left(B^{\prime}\right)\right) d y+\int_{\epsilon}^{c} \phi(\mu(A)) d y \\
& =\epsilon \phi\left(\mu(A)+\mu\left(B^{\prime}\right)\right)+(c-\epsilon) \phi(\mu(A)) .
\end{aligned}
$$

But since there exists $\alpha>\mu(A)$ so that $\phi$ is not constant in any subinterval of the interval $[0, \alpha]$, it follows that $\phi\left(\mu(A)+\mu\left(B^{\prime}\right)\right)>\phi(\mu(A))$. Therefore

$$
\left\|f_{i}\right\|>\epsilon \phi(\mu(A))+(c-\epsilon) \phi(\mu(A))=1,
$$

contradicting $f_{1}, f_{2} \in S\left(N_{\phi}(E)\right)$. Thus $f_{1} \chi_{A} \neq f_{2} \chi_{A}$, and it can be assumed that $\left\{x \in X:\left\|f_{i}(x)\right\|>0\right\} \subseteq A$. Now

$$
1=\left\|f_{i}\right\|=\int_{0}^{\infty} \phi\left(\lambda_{f_{i}}(y)\right) d y=\int_{0}^{\infty} \phi\left(\frac{\lambda_{f_{i}}(y)}{\mu(A)} \mu(A)\right) d y .
$$

But $\left\{x \in X:\left\|f_{i}(x)\right\|>0\right\} \subset A$ implies that $\lambda_{f_{i}}(0) \leqslant \mu(A)$ so $\lambda_{f_{i}}(y) \leqslant \mu(A)$ for all $y \geqslant 0$. Thus $\lambda_{f_{i}}(y) / \mu(A) \leqslant 1$ and therefore

$$
\begin{aligned}
\int_{0}^{\infty} \phi\left(\frac{\lambda_{f_{i}}(y)}{\mu(A)} \mu(A)\right) \cdot d y & \geqslant \int_{0}^{\infty} \frac{\lambda_{f_{i}}(y)}{\mu(A)} \phi(\mu(A)) d y=\frac{\phi(\mu(A))}{\mu(A)} \int_{0}^{\infty} \lambda_{f_{i}}(y) d y \\
& =\frac{\phi(\mu(A))}{\mu(A)} \int_{0}^{\infty} \lambda_{\tilde{f}_{i}}(y) d y=\int_{0}^{\infty} m\left\{x \in X: \tilde{f}_{i}(x)>y\right\} d y \\
& =\int_{X} \widetilde{f}_{i}(x) d m(x)=1 .
\end{aligned}
$$

Therefore

$$
\int_{0}^{\infty}\left[\phi\left(\lambda_{f_{i}}(y)\right)-\frac{\phi(\mu(A))}{\mu(A)} \lambda_{f_{i}}(y)\right] d y=0,
$$

and since the integrand is $\geqslant 0$, this implies that

$$
\phi\left(\lambda_{f_{i}}(y)\right)=\frac{\phi(\mu(A))}{\mu(A)} \lambda_{f_{i}}(y) \quad \text { for } y \text {-a.e. }
$$

Next it is shown that either $\lambda_{f_{1}}$ or $\lambda_{f_{2}}$ has a range of at least two positive values. That is, there exist $y_{1}, y_{2}, y_{1}<y_{2}$, such that either $\lambda_{f_{1}}\left(y_{1}\right)>\lambda_{f_{1}}\left(y_{2}\right)>$ 0 or $\lambda_{f_{2}}\left(y_{1}\right)>\lambda_{f_{2}}\left(y_{2}\right)>0$. If this is not true, then, for some $\alpha_{1}, \alpha_{2}>0$, $\lambda_{f_{i}}(y)=0$ or $\lambda_{f_{i}}(y)=\alpha_{i}$ for all $y \geqslant 0$. Thus, by Lemma $9\left\|f_{i}(x)\right\|=k_{i} \chi_{A_{i}}(x)$ with $A_{i} \subseteq A$. But Lemma 11 implies that either $\phi(x)=k x$ for $x \in[0, \mu(A)]$ or $k_{1}=k_{2}=c$ and $A_{1}=A_{2}=A$. In both cases this is a contradiction, and there- 
fore there exists such a pair $y_{1}, y_{2}$ for $f_{1}$ or $f_{2}$. Thus it may be assumed that $\lambda_{f_{1}}\left(y_{1}\right)>\lambda_{f_{1}}\left(y_{2}\right)>0$.

Let

$$
B=\left\{y \geqslant 0: \phi\left(\lambda_{f_{1}}(y)\right)=\phi(\mu(A)) \lambda_{f_{1}}(y) / \mu(A)\right\}
$$

so that $B$ has Lebesgue measure zero. Since $\lambda_{f_{1}}(0) \leqslant \mu(A)<\infty$, Lemma 10 may be applied to conclude that there exist $y_{1}^{\prime}, y_{2}^{\prime} \in[0, \infty) \sim B$ such that $\lambda_{f_{1}}\left(y_{1}^{\prime}\right)>\lambda_{f_{1}}\left(y_{2}^{\prime}\right)$ $>0$. Thus

$$
\phi\left(\lambda_{f_{1}}\left(y_{i}^{\prime}\right)\right)=\frac{\phi(\mu(A))}{\mu(A)} \lambda_{f_{1}}\left(y_{i}^{\prime}\right)
$$

By Proposition 1,

$$
\phi(x)=\phi(\mu(A)) x / \mu(A)
$$

for $x \in[0, \mu(A)]$ and this is a contradiction. Therefore $f \in \operatorname{Ext} U\left(N_{\phi}(E)\right)$.

PROOF OF Proposition 12. It is first shown that if $f \in \operatorname{Ext} U\left(N_{\phi}(E)\right)$, then $A$ is an atom of $(X, \Sigma, \mu)$. Suppose $A$ is not an atom. Then $A=A_{1} \cup A_{2}$, $\mu\left(A_{i}\right)>0$ and $A_{1} \cap A_{2}=\varnothing$. Choose $\epsilon_{1}, \epsilon_{2}>0$ such that

$$
\epsilon_{1} / \epsilon_{2}=\mu\left(A_{2}\right) / \mu\left(A_{1}\right) \text { and } \max \left(\epsilon_{1}, \epsilon_{2}\right)<c \text {. }
$$

Define

$$
f_{1}=\left(1+\epsilon_{1} / c\right) f \chi_{A_{1}}+\left(1-\epsilon_{1} / c\right) f \chi_{A_{2}}
$$

and

$$
f_{2}=\left(1-\epsilon_{1} / c\right) f \chi_{A_{1}}+\left(1-\epsilon_{1} / c\right) f \chi_{A_{2}} .
$$

Then $f=\left(f_{1}+f_{2}\right) / 2$ and $f_{1} \neq f_{2}$.

$$
\left\|f_{1}\right\|=\left(c-\epsilon_{2}\right) \phi(\mu(A))+\left(\epsilon_{1}+\epsilon_{2}\right) \phi\left(\mu\left(A_{1}\right)\right)=k c \mu(A)=1 .
$$

Similarly it is verified that $\left\|f_{2}\right\|=1$. Thus a contradiction obtains. Thus $A$ is an atom so that $f(x)=x_{0} \chi_{A}(x)$ with $\left\|x_{0}\right\|=c$. From this it follows easily that $x_{0} /\left\|x_{0}\right\| \in \operatorname{Ext} U(E)$.

By Proposition 9, it is enough to show that if $f(x)=x_{0} \chi_{A}(x)$ with $A$ an atom and $x_{0} /\left\|x_{0}\right\| \in$ Ext $U(E)$ and either $\mu(X \sim A)=0$ or $\mu(A)$ satisfies (*), then $f \in \operatorname{Ext} U\left(N_{\phi}(E)\right)$. If $\mu(X \sim A)=0$, then $X$ is an atom and $f(x)=x_{0}$ is easily seen to be an extreme point. Assume that $\mu(X \sim A)>0$, and $f=$ $\left(f_{1}+f_{2}\right) / 2$ with $f_{1} \neq f_{2}$ and $f_{1}, f_{2} \in S\left(N_{\phi}(E)\right)$. Then

$$
f_{1}(x)=x_{1} \chi_{A}(x)+g(x) \text { and } f_{2}(x)=x_{2} \chi_{A}(x)-g(x)
$$

with

$$
\lambda_{g}(0) \leqslant \mu(X-A), \quad x_{1}+x_{2}=2 x_{0}, \quad \text { and }\|g\|>0 .
$$

Choose $y_{0}>0$ such that $\lambda_{g}\left(y_{0}\right)>0$. For convenience, assume that $y_{0}<$ $\min \left(\left\|x_{1}\right\|,\left\|x_{2}\right\|\right)$. Then 


$$
\begin{aligned}
1= & \left\|f_{1}\right\|=\int_{0}^{\infty} \phi\left(\lambda_{f_{1}}(y)\right) d y=\int_{0}^{y_{0}} \phi\left(\mu(A)+\lambda_{g}(y)\right) d y \\
& +\int_{y_{0}}^{\left\|x_{1}\right\|} \phi\left(\mu(A)+\lambda_{g}(y)\right) d y+\int_{\left\|x_{1}\right\|}^{\infty} \phi\left(\lambda_{g}(y)\right) d y \\
& \geqslant \int_{0}^{y_{0}} \phi\left(\mu(A)+\lambda_{g}(y)\right) d y+\int_{y_{0}}^{\left\|x_{1}\right\|} \phi(\mu(A)) d y \\
& \geqslant \int_{0}^{y_{0}} \phi\left(\mu(A)+\lambda_{g}\left(y_{0}\right)\right) d y+\left(\left\|x_{1}\right\|-y_{0}\right) \phi(\mu(A)) \\
& =y_{0}\left(\phi\left(\mu(A)+\lambda_{g}\left(y_{0}\right)\right)\right)+\left(\left\|x_{1}\right\|-y_{0}\right) \phi(\mu(A)) \\
> & y_{0} \phi(\mu(A))+\left(\left\|x_{1}\right\|-y_{0}\right) \phi(\mu(A))=\left\|x_{1}\right\| \phi(\mu(A)),
\end{aligned}
$$

the last inequality following from the assumption that there exists $\alpha>\mu(A)$ such that $\phi$ is not constant in any subinterval of the interval $[0, \alpha]$. Similarly $1=$ $\left\|f_{2}\right\|=\int_{0}^{\infty} \phi\left(\lambda_{f_{2}}(y)\right) d y>\left\|x_{2}\right\| \phi(\mu(A))$. It follows that

$$
2=\left\|f_{1}\right\|+\left\|f_{2}\right\| \geqslant\left\|x_{1}+x_{2}\right\| \phi(\mu(A))=2,
$$

a contradiction, completing the proof of the proposition.

The following theorem summarizes the preceding propositions, and provides a characterization of extreme points of $N_{\phi}(E)$. If $f \in N_{\phi}(E), \widetilde{f}: X \rightarrow R$ is the function $\tilde{f}(x)=\|f(x)\|$.

THEOREM 5. Let $f \in S\left(N_{\phi}(E)\right)$. Let $A=$ Support of $f$ :

(i) If $\mu(A)=\infty, f \in \operatorname{Ext} U\left(N_{\phi}(E)\right)$, if and only if $\mu(X \sim A)=0, \tilde{f} \in$

Ext $U\left(N_{\phi}(R)\right)$, and $P f \in \operatorname{Ext} C$.

(ii) If $\mu(A)<\infty$, then

(a) if there is a $k>0$ such that $\phi(x)=k x$ for $x \in[0, \mu(A)]$, then $f \in \operatorname{Ext} U\left(N_{\phi}(E)\right)$ if and only if $f(x)=x_{0} \chi_{A}(x)$, with $x_{0} /\left\|x_{0}\right\| \in \operatorname{Ext} U(E)$, and $\tilde{f} \in \operatorname{Ext} U\left(N_{\phi}(R)\right)$,

(b) if there does not exist such a $k, f \in \operatorname{Ext} U\left(N_{\phi}(E)\right)$ if and only if $\tilde{f} \in \operatorname{Ext} U\left(N_{\phi}(R)\right)$, and $P f \in \operatorname{Ext} C$.

The characterization provided in the preceding theorem is not complete since in order to check whether a given function $f$ is an extreme point of $U\left(N_{\phi}(E)\right)$, among others, one has to check whether it is of the form $\left(f_{1}+f_{2}\right) / 2$ where $\left\|f_{i}(x)\right\|=c \chi_{A}(x), f_{1} \neq f_{2}$. However if $E$ is a separable Banach space a complete characterization of extreme points of $U\left(N_{\phi}(E)\right)$ could be deduced from the preceding theorem. The technique is to use a measurable selection theorem for set valued functions, and is similar to the proofs of Theorem 2, in Sundaresan [28], and Proposition 1 in Johnson [9 $]$. 
THEOREM 6. Let $E$ be a separable Banach space, and $(X, \Sigma, \mu)$ be as in the preceding theorem. Let $\mu$ be a complete measure. Then a function $f \in$ Ext $U\left(N_{\phi}(E)\right)$ if and only if (1) $\tilde{f} \in \operatorname{Ext} U\left(N_{\phi}(R)\right)$, and (2) $f(x) /\|f(x)\| \in$ Ext $U(E)$ for $x$, $\mu$-a.e. in the support of $f$.

Proof. If $f$ satisfies (1) and (2) applying Theorem 4, $\S 6$, and definition of $P f$, it is at once verified that $\|f(x)\|=c \chi_{A}(x)$ where $A=$ Support of $f$, and $c$ is a positive number, and $P f \in \operatorname{Ext} C$. Hence $f \in \operatorname{Ext}\left(N_{\phi}(E)\right)$ as a consequence of Theorem 5 .

Conversely if $\tilde{f} \in \operatorname{Ext} U\left(N_{\phi}(E)\right)$ then from Theorem 5, $f \in \operatorname{Ext}\left(N_{\phi}(R)\right)$, and $P f \in$ Ext $C$. Now from Corollary 2, [9, $]$, it is at once verified that $f(x) /\|f(x)\| \in \operatorname{Ext} U(E)$, for $x, \mu$-a.e. in the support of $f$.

\section{REFERENCES}

1. S. Banach, Théorie des opérations linéaires, Monografie Mat., PWN, Warsaw, 1932; reprint, Chelsea, New York, 1955. MR 17, 175.

2. P. Billingsley, Convergence of probability measures, Wiley, New York, 1968. MR 38 \#1718.

3. M. M. Day, Normed linear spaces, 2nd rev. ed., Academic Press, New York; Springer-Verlag, Berlin, 1962. MR 26 \#2847.

4. N. Dinculeanu, Vector measures, Internat. Ser. of Monographs in Pure and Appl. Math., vol. 95, Pergamon Press, Oxford; VEB Deutscher Verlag, Berlin, 1967. MR 34 \#6011b.

5. N. Dunford and J. T. Schwartz, Linear operators. I: General theory, Pure and Appl. Math., vol. 7, Interscience, New York, 1958. MR 22 \#8302.

6. P. R. Halmos, Measure theory, Van Nostrand, Princeton, N. J., 1950. MR 11, 504.

7. I. Halperin, Function spaces, Canad. J. Math. 5 (1953), 273-288. MR 15, 38.

8. G. H. Hardy, J. E. Littlewood and G. Pólya, Inequalities, 2nd ed., Cambridge Univ. Press, New York, 1952. MR 13, 727.

9. R. A. Hunt, An extension of the Marcinkiewicz interpolation theorem to Lorentz spaces, Bull. Amer. Math. Soc. 70 (1964), 803-807; Addendum, ibid. 71 (1965), 396. MR 29 \#6292; 30 \#2331. $107-112$.

$9_{1}$. J. A. Johnson, Extreme measurable selections, Proc. Amer. Math. Soc. 44 (1974),

$9_{2}$. E. De Jonge, $A$ pair of mutually associated Banach function spaces, Indag. Math. 77 (1974), 148-156.

10. M. A. Krasnosel'skil and Ja. B. Rutickil, Convex functions and Orlicz spaces, Problems of Contemporary Mathematics, GITTL, Moscow, 1958; English transl., Noordhoff, Groningen, 1961. MR 21 \#5144; 23 \#A4016.

11. L. E. Leonard and K. Sundaresan, Smoothness and duality in $L_{p}(E, \mu)$, J. Math. Anal. Appl. 46 (1974), 513-522. MR 11, 442.

12. G. G. Lozentz, Some new functional spaces, Ann. of Math. (2) 51 (1950), 37-55.

13. - On the theory of spaces $\Lambda$, Pacific J. Math. 1 (1951), 411-429. MR 13, 470.

14. - Relations between function spaces, Proc. Amer. Math. Soc. 12 (1961), 127-132. MR 23 \#A511.

15. G. G. Lorentz and T. Shimogaki, Interpolation theorems for the pairs of spaces $\left(L^{p}, L^{\infty}\right)$ and $\left(L^{1}, L^{q}\right)$, Trans. Amer. Math. Soc. 159 (1971), 207-221.

16. W. A. J. Luxemburg, Rearrangement-invariant Banach function spaces, Queen's Papers in Pure and Appl. Math., no. 10, Queen's University, Kinston, Ont., 1967, pp. 83-144. 
17. W. A. Luxemburg, Riesz spaces, North-Holland, Amsterdam, 1971.

18. R. O'Neil, Convolution operators and $L(p, q)$ spaces, Duke Math. J. 30 (1963), 129-142. MR 26 \#193.

19. R. R. Phelps, Dentability and extreme points J. Functional Anal. 17 (1974), 78-90.

20. M. A. Rieffel, The Radon-Nikodym theorem for the Bochner integral, Trans. Amer. Math. Soc. 131 (1968), 466-487. MR 36 \#5297.

21. J. V. Ryff, Orbits of $L^{1}$-functions under doubly stochastic transformations, Trans. Amer. Math. Soc. 117 (1965), 92-100. MR 35 \#762.

22. W. L. C. Sargent, Some sequence spaces related to the $l^{p}$ spaces, J. London Math. Soc. 35 (1960), 161-171. MR 22 \#7001.

23. E. M. Semenov, Imbedding theorems for Banach spaces of measurable functions, Dokl. Akad. Nauk SSSR 156 (1964), 1292-1295 = Soviet Math. Dokl. 5 (1964), 831-834. MR 30 \#3368.

24. M. S. Steigerwalt and A. J. White, Some function spaces related to $L_{p}$ spaces, Proc. London Math. Soc. (3) 22 (1971), 137-163. MR 43 \#5304.

25. E. M. Stein and G. Weiss, Introduction to Fourier analysis on Euclidean spaces, Princeton Math. Ser., no. 32, Princeton Univ. Press, Princeton, N. J., 1971. MR 46 \#102.

26. H. Steinhaus, Sur la probabilité de la convergence des séries, Studia Math. 2 (1930), 21-39.

27. K. Sundaresan, Extreme points of the unit cell in Lebesgue-Bochner function spaces. I, Proc. Amer. Math. Soc. 23 (1969), 179-184; Errata, ibid. 25 (1970), 922. MR 40 \#719; 41 \#4222.

28. - Extreme points of the unit cell in Lebesgue-Bochner function spaces, Colloq. Math. 22 (1970), 111-119. MR 43 \#2493.

29. S. L. Trojanski, On locally uniformly convex and differentiable norms in certain non-separable Banach spaces, Studia Math. 37 (1970/71), 173-180. MR 46 \#5995.

30. A. Zygmund, Trigonometric series. Vols. I, II, Cambridge Univ. Press, London, 1968. MR 38 \#4882.

DEPARTMENT OF MATHEMATICS, UNIVERSITY OF WYOMING, LARAMIE, WYOMING 82071 